\title{
Min-max Game Theory for Elastic and Visco-Elastic Fluid Structure Interactions
}

\author{
Irena Lasiecka ${ }^{1, *}$, Roberto Triggiani ${ }^{1}$ and Jing Zhang ${ }^{2}$ \\ ${ }^{I}$ Department of Mathematics, University of Virginia, Charlottesville, VA 22901, USA \\ ${ }^{2}$ Department of Mathematics and Computer Science, Virginia State University, St Petersburg, VA, USA
}

\begin{abstract}
We present the salient features of a min-max game theory developed in the context of coupled PDE's with an interface. Canonical applications include linear fluid-structure interaction problem modeled by Oseen's equations coupled with elastic waves. We shall consider two models for the structures: elastic and visco-elastic. Control and disturbance are allowed to act at the interface between the two media. The sought-after saddle solutions are expressed in a pointwise feedback form, which involves a Riccati operator; that is, an operator satisfying a suitable non-standard Riccati differential equation. Motivations, applications as well as a brief historical account are also provided.
\end{abstract}

Keywords: Elasticity, fluid-structure interactions, min-max game problem, Riccati operator, visco-elasticity.

\section{INTRODUCTION}

\subsection{General Comments}

We consider a min-max game problem as formulated for coupled PDE control systems arising in fluid structure interaction models. We shall consider two drastically different models for the structure: elastic and viscoelastic. While the first prevails in engineering applications when the solid is immersed into a fluid, the second viscoelastic model is often used in biological-medical applications where the structure has some viscoelastic response.

The purpose of this article is to present general abstract results in the area of game theory and the associated feedback synthesis via a non-standard Riccati equation along with their applications to the fluid structure interactions mentioned above. One should stress at the outset that while game theory was established by John von Neumann [1] and later developed by J. Nash, Isaacs and others [2-7, 5], it was a work of Basar and Bernhard [8] that has had influence on this theory developed for dynamics governed by abstract ODE's and PDE's. An extensive, yet not complete list is given in $[9,10]$. It suffices to say that the theory has been considerably developed in the context of linear semigroups with bounded control actions and bounded disturbances. However, the presence of unbounded controls-disturbances provides for a main novelty and challenge of the problem under consideration in this article. On the other hand, applications such as they arise in the context of coupled PDE systems, where the control/disturbance is typically active at

*Address correspondence to this author at the Department of Mathematics, University of Virginia, Charlottesville, VA 22901, USA;

Tel: 434-924-8896; E-mail: i12v@virginia.edu the interface (hence on a manifold of lower dimension), -lead invariably to unbounded controls -disturbances formulation of the problem. These applications have provided the impetus for recent developments in abstract variational analysis. This now requires an arsenal of new tools enabling one to deal with unbounded actuation-disturbances control problems.

It is the purpose of this article to give an overview of recent abstract results and put these in the context of physical applications. We shall show how to apply abstract theory, how to verify theoretical assumptions, imposed by that theory, on a specific class of models of interest. Finally, we shall interpret game theory results in the context of boundary controlled fluid structure interactions. It turns out that type of elastic or viscoelastic response of the structural material has significant implications on the final game theoretic results claimed.

The main aim of this article is to present a complete mini-max theory in the context of models consisting of coupled parabolic - parabolic dynamics with control action occurring on the interface between two different environments (fluid and structure). As we shall see, this setup brings forward new mathematical challenges.

\subsection{Game Theory for a Fluid-Structure Interaction Model: Engineering and Biological Motivations}

The fluid-structure interaction model is well established in both mathematical and engineering literatures $[11,12]$ and the applications range from naval and aerospace engineering to cell biology and biomedical engineering [12-17]. In order to motivate a reader we shall describe some of them. It is important to note that depending on the application considered the model of structure or solid may be purely elastic or visco-elastic. 


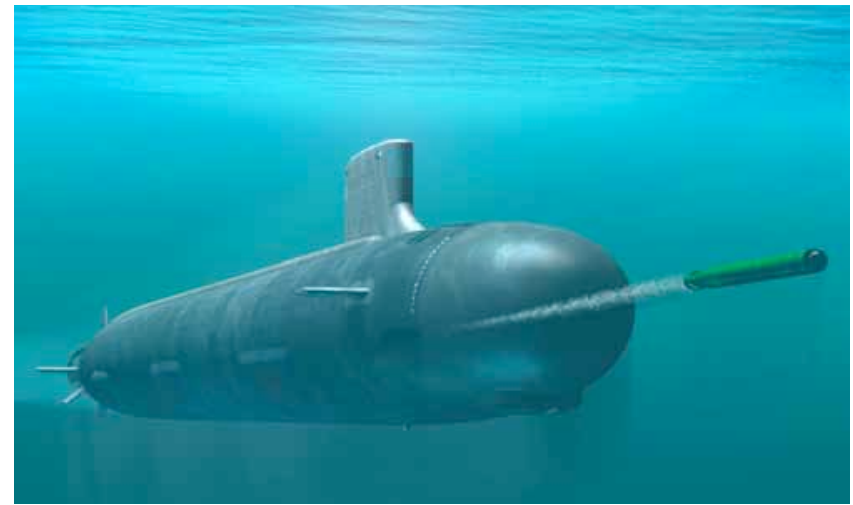

Fig. (1). Submarine immersed in water.

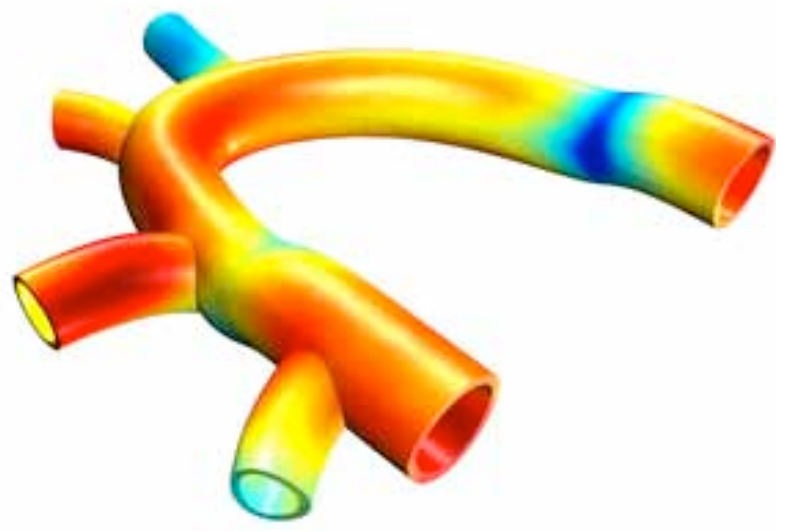

Fig. (2). Blood cells surrounded by blood plasma.

1. One interesting application of this model is the measurement of the dosage of antibiotics prescribed to a patient. The research carried out in this area has raised wide attention as prescription drug dosage has become a fast growing problem recent years. It is documented that in 2008, a total of 36,450 deaths were attributed to drug overdose, and this number is still increasing. Our PDE model provides a mathematical approach for determining the optimal dosage for the antibiotics. Here the "optimal dosage" is defined as the dosage that gives the desired effect with minimum side effects. We work under the assumption that doctors always want to minimize the antibiotics dosage prescribed to patients (so as to minimize the side effects). While on the other hand, if the dosage is too low, the antibiotics can not kill the bacteria; worse still, bacteria can even get antibioticresistant. In this scenario, the antibiotics and the bacteria become two players in a noncooperative game. Our research targets the goal of finding the minimum antibiotics dosage that kills the worst bacteria in the patient, thus cures the bacteria infection completely. This is a typical minimax algorithm in game theory. In the PDE model, the effects of the antibiotics and the bacteria to the patient is described by the control and the disturbance respectively.

Our objective is to develop the feedback optimal dosage for the antibiotics based on the solution (the feedback operator) of a certain Riccati equation. It is known that the Riccati theory is a very powerful tool in designing and computing feedback controls in the linear quadratic regulator problems. In our study, we extend the classic Riccati equations to so called non-standard Riccati equations. The difference between a non-standard Riccati equation and the

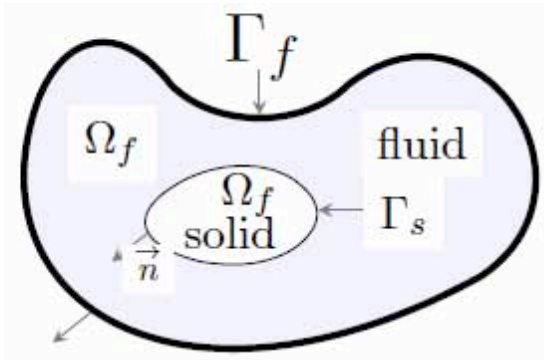

Fig. (3). An elastic body immersed inside a fluid.

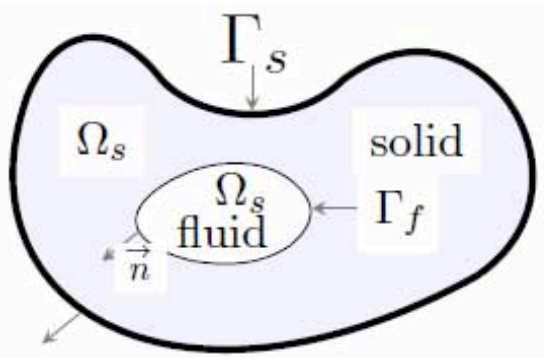

Fig. (4). A fluid surrounded by an elastic wall.

usual Riccati equation is an extra term corresponding to the present disturbance in the PDE system, which, however, occurs with a "bad" (negative) sign in front. The construction of the non-standard Riccati equation associated to the PDE model provides us with a feedback algorithm for computing the optimal antibiotic dosage for a particular patient due to his physical conditions, which can be monitored by his blood test, such as the white blood cells counts, etc; whereas our PDE model describes the blood status by the interaction between the blood cells (modeled by the equation of the system of elasticity that account for viscoelastic effects) and the blood plasma (modeled by the linearized Navier-Stokes equation Fig. (2)).

2. Another medical application involves tomography and ultrasound techniques where the goal is a detection of a solid within the cavity filled with body fluids. The actuation is in a form of a force (laser force) applied to the interface.

3. A classical example of engineering application is a solid -elastic body submersed in a water (submarine Fig. 1) . The goal here is to control vibrations of the elastic body or else minimizing signature of the solid. This is done on the boundary of the solid by applying frictional or viscous dampers (layered material) to the surface of the body. In this case, the body is modeled by the dynamic system of elasticity that may ignore visco-elastic effects.

\section{THE FLUID-STRUCTURE INTERACTION - PDE MODEL}

\subsection{Description of the Model}

In what follows we shall consider fluid-structure interaction model corresponding to the situation where the elastic body is immersed inside the fluid (Fig. 3). Other configurations that correspond to a fluid surrounded by elastic walls (blood aortas -Fig. 2) can also be considered in the same manner. In this latter case the fluid constitutes the internal domain while the structure-walls are on the exterior. See Fig. (4) below. We shall focus in this paper on the first 
configuration. The assumptions made is that the oscillations of the body are rapid but small. This allows to neglect the dynamics of the interface [12].

Let $\Omega \in \mathrm{R}^{n}, \quad n=2,3$ be an open bounded domain consisting of an interior region $\Omega_{s}$ and an exterior region $\Omega_{f}$, see picture below. We denote by $\Gamma_{f}$ the outer boundary of the domain while $\Gamma_{s}$ is the boundary of region $\Omega_{s}$ which also borders the exterior region $\Omega_{f}$ and where the interaction of the two systems take place. Thus $\Gamma_{s}$ is the interface between the two media. Let $u$ be an $n$ dimensional vector function defined on $\Omega_{f}$ representing the velocity of the fluid, while the scalar function $p$ represents the pressure. Additionally, let $v$ and $v_{t}$ be the $\mathrm{n}$ dimensional displacement and velocity functions of the solid $\Omega_{s}$. We also denote by $v$ the unit normal vector outward with respect to the domain $\Omega_{f}$. The boundaryinterface control is represented by $g \in L_{2}\left(0, T ;\left(L_{2}\left(\Gamma_{s}\right)\right)^{n}\right)$ and is active on the boundary $\Gamma_{s}$. For simplicity (but it also seems a physically reasonable situation), we first assume that the deterministic disturbance $w=\left\{w_{1}, w_{2}\right\}$ acts with one component $w_{1}$ in $\Omega_{f}$ and one component $w_{2}$ in $\Omega_{s}$. We work under the assumption of small but rapid oscillations of the solid body, so that the interface $\Gamma_{s}$ may be assumed to be static, see [12] for more modeling details. Additional reference to the non-linear model of the Navier-Stokes equations include [11], p126, which, in turn, makes reference to a biological model [18]. The correct model of a moving structure immersed in a Navier-Stokes fluid (say a boat that drifts under the action of the fluid; not a self-propelled structure) appears to be still unresolved. A discussion leading to an arbitrary Lagrange-Euler formulation is given in [19], equation (8.6), p219. A more recent effort is in [20].

\subsection{Boundary Control with Boundary/Interior Disturbance PDE Model}

We shall consider a model resulting from the coupling of Osseen's operator (fluid) with dynamic system of elasticity. The coupling is on interface between the two different environments.

Given the boundary control $g=\left(g_{0}, g_{1}\right) \in L_{2}(0, T ; \mathrm{U})$ with $\left.\mathrm{U}=\left(H^{1 / 2}\left(\Gamma_{s}\right)\right)^{n} \times\left(L_{2}\left(\Gamma_{s}\right)\right)^{n}\right)$ at the interface, and the interior deterministic disturbance $w=\left\{w_{1}, w_{2}, w_{3}\right\} \in L_{2}(0, T ; \mathrm{V})$ where $\left.\left.\mathrm{V}=\left(L_{2}\left(\Omega_{f}\right)\right)^{n} \times\left(L_{2}\left(\Omega_{s}\right)\right)^{n}\right) \times L_{2}\left(\Gamma_{s}\right)^{n}\right)$, we consider the following parabolic-hyperbolic coupled system of partial differential equations which couples Oseen's equation (fluid) with dynamic system of elasticity (resp. viscoelasticity). The unknowns are $\left\{u, v, v_{t}, p\right\}$, describing fluid velocity $u$, solid's displacement $v$, solid's velocity $v_{t}$ and the pressure $p$.

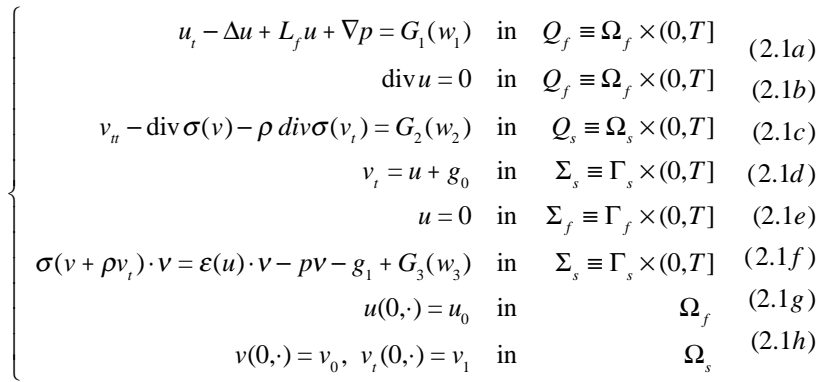

The elastic strain tensor $\varepsilon$ and the stress tensor $\sigma$, are $n \times n$ symmetric matrices respectively given by

$\varepsilon_{i j}(u)=\frac{1}{2}\left(\frac{\partial u_{i}}{\partial x_{j}}+\frac{\partial u_{j}}{\partial x_{i}}\right)=\varepsilon_{j i}(u)$

and

$$
\begin{aligned}
& \sigma(u)=\lambda \operatorname{tr} \varepsilon(u) I+2 \mu \varepsilon(u)=\left(\sigma_{i j}(u)\right)_{i, j=1}^{n} \\
& \sigma_{i j}(u)=\lambda\left(\sum_{k=1}^{n} \varepsilon_{k k}(u)\right) \delta_{i j}+2 \mu \varepsilon_{i j}(u)=\sigma_{j i}(u)
\end{aligned}
$$

where $\rho \geq 0, \lambda>0$ and $\mu>0$ are the Lamé constants. Obviously we have

$|\varepsilon(u)| \leq|\nabla u| ;|\sigma(u)| \leq 2 \max \{\lambda, 2 \mu\}|\varepsilon(u)| \leq 2 \max \{\lambda, 2 \mu\}|\nabla u|$ (2.4)

The boundary conditions in (2.1f) represent balance of the stresses, while condition (2.1d) represents matching of the velocities between the fluid and the solid.

The constant $\rho \geq 0$ plays an important role. When $\rho>0$ the model of the structure accounts for visco-elastic effects a typical situation when modeling blood passing through the arteries [13, 14].

The case of a pure elastic solid (such as submarine immersed in the water) corresponds to $\rho>0$. From the mathematical point of view, the two models are vastly different. The first one $(\rho>0)$ corresponds to a parabolicanalytic coupling of dynamics, while the second case $(\rho=0)$ corresponds to parabolic-hyperbolic coupling. We shall see later that this fact has implications on the results obtained for each model.

The term $L_{f} u$ is a linearization of the convective term of the Navier-Stokes term $(u \cdot \nabla) u$ and is defined as

$$
L_{f} u=(u \cdot \nabla) y_{e}+\left(y_{e} \cdot \nabla\right) u, \quad \operatorname{div} y_{e}=0,\left.\quad y_{e}\right|_{\Gamma_{f}}=0
$$

where $y_{e}$ is a steady-state or equilibrium solution forced by an external force $f \in L_{2}(\Omega)$; that is, a time independent smooth vector function in $\left[H^{2}\left(\Omega_{f}\right)\right]^{n}$ with the properties: $\operatorname{div} y_{e}=0,\left.y_{e}\right|_{\Gamma_{f}}=0$.

The disturbance operators $G_{1}, G_{2}, G_{3}$ are assumed linear. They describe location and intensity of the internal disturbances affecting the fluid $G_{1}\left(w_{1}\right)$ and the solid $G_{2}\left(w_{2}\right)$. The third disturbance $G_{3}\left(w_{3}\right) \in L_{2}\left(\Sigma_{f}\right)$ is located at the interface between the two regions. 
The control functions $g_{0}$ and $g_{1}$ act on the boundary interface between the two regions. Our goal is to select suitable boundary-control mechanisms $g_{0}, g_{1}$, expressed in the feedback form as a functions of the state variable $\left(u(t), v(t), v_{t}(t)\right)$, that will provide optimal response to the system in the presence of disturbances.

\subsection{Mini-max Optimization Problem}

Let $\mathrm{U}$ (resp. $\mathrm{V}$ ) be given as above. With $T>0$ preassigned, control functions $g=\left(g_{0}, g_{1}\right) \subset L_{2}(0, T ; \mathrm{U})=L_{2}(\mathrm{U})$ and disturbances $w=\left(w_{1}, w_{2}, w_{3}\right) \in L_{2}(\mathrm{~V})$ consider the quadratic cost functional

$$
J(y, g, w)=\int_{0}^{T}\left(|g(t)|_{U}^{2}+|\mathrm{R} y(t)|_{Z}^{2}\right) d t-\gamma^{2} \int_{0}^{T}|w(t)|_{V}^{2} d t
$$

Here the state variable $y(t)=\left(u(t), v(t), v_{t}(t)\right) \in \mathrm{H}$ satisfies (2.1) for a given control $g$ and disturbance $w$. Hilbert space $\mathcal{H}$ represents standard finite energy space associated with the physical model representing fluid and waves (to be introduced later) and the observation $\mathrm{R} \in L(\mathrm{H} \rightarrow \mathrm{Z})$ with a given output Hilbert space $\mathcal{Z}$. The min-max game problem corresponding to (2.6) is

$\sup _{w \in L_{2}(\mathrm{~V})} \inf _{g \in L_{2}(\mathrm{U})} J(y, g, w)=J\left(y^{*}, g^{*}, w^{*}\right)$

Our aim is to find state-feedback representation for both controls and disturbances

$g^{*}(t)=K(t) y^{*}(t), \quad w^{*}(t)=D(t) y^{*}(t), t \in(0, T)$

where the feedback operators $K(t)$ and $D(t)$ are bounded. This is to say:

$$
K(t) \in L(\mathrm{H} \rightarrow \mathrm{U}), D(t) \in L(\mathrm{H} \rightarrow \mathrm{V}), t \in[0, T]
$$

We note that the requirement that the state feedback be bounded (very practical engineering requirement) provides for mathematical challenge of the problem. It is well known that the "unbounded controls-disturbances " -such as point or boundary controls - while still yielding well defined open loop controls, lead to unbounded state feedback representations in the closed loop formulation, see [21-23] and references therein. Thus, in order to have bounded state feedback representation in the context of interface controls some "special" structure needs to be discovered. It is the aim of this work to reveal rather special structure of the physical system ("singular estimate property") which is responsible for well behaving state feedbacks to be later constructed by Riccati theory.

While mini-max game theory problem has been considered in the past literature-both abstractly and also concretely in the context of fluid structure interactions [24, 25], this is the first time when viscoelastic structure is considered and Dirichlet type boundary controls (functions $g_{0}$ ) are employed. In fact, while Neumann boundary controls, due to higher regularity and variational nature of the formulation [11] are more amenable to the mathematical treatment, physically attractive Dirichlet controls invariably lead to much more challenging analysis (they do not reside in a natural energy space). Dirichlet boundary control problem for fluid structure models, even in the context of classical Riccati Equations, has been open and considered difficult for some time. Thus, this is the first manuscript where this open problem is being addressed and solved.

The outline of the paper is as follows. In section 3 we recall general mini-max theory results that were recently obtained for abstract systems with unbounded controlsdisturbances. In section 4 we shall represent fluid-structure interaction model as an abstract control system. The main result is stated in Theorem 3.1, Section 4. Section 5 is devoted to the proofs. Here the main point is to verify the abstract assumptions imposed by the general theory of section 3. This will allow for application of such unified treatment. The paper concludes with conclusions and open questions.

\section{MIN-MAX GAME THEORY - GENERAL THEORY}

\subsection{General Setup and Overview}

To study the min-max game theory for parabolichyperbolic type coupled PDEs, our main difficulty is the construction of the associated non-standard Riccati equation in the context of boundary control/boundary disturbance. The latter yields a mathematically challenging analysis (in the setting of "highly unbounded" operators), where the standard approach (within the setting of "bounded" operator) fails. This is due to the fact that the feedback operator along with the associated coefficents in the non-standard Riccati equation may not be well defined. The intrinsic reason for this is the lack of sufficient regularity in the PDE system. The theoretical framework of constructing the Riccati equation associated with systems generating analytic semigroups has indeed been established [9, 10, 26], Chapter 6; whereas for each of these parabolic-hyperbolic coupled PDE models, the overall dynamics may not be smooth enough due to the presence of the hyperbolic component. Nevertheless, the parabolic component does impose a partial smoothing on the overall dynamical evolution, which is captured in the "singular estimate" property. This property is critical for the development and validity of mini-max Riccati theory in the case of unbounded actions or unbounded coefficients. We shall report below the main results which are pertinent to this study. Paper [24] consider the following abstract dynamics:

$$
\text { Abstract Model: }\left\{\begin{array}{l}
y(t)=A y(t)+B g(t)+G w(t) \text { on }\left[\mathrm{D}\left(A^{*}\right)\right] \\
y(0)=y_{0} \in Y
\end{array}\right.
$$

where $\{y(t), g(t), w(t)\}$ represent the state, control and disturbance respectively, $A$ is the generator of a strongly continuous semigroup on the Hilbert state space $Y, B$ is a linear, highly unbounded operator over the control space $U$, such that $B: U \rightarrow\left[\mathrm{D}\left(A^{*}\right)\right]$ or $A^{-1} B \in \mathrm{L}(U ; Y)$, similarly for $G$ over the disturbance space $V$. Moreover, both satisfy the following "singular estimate" at the origin:

$$
\begin{aligned}
& \left|e^{A t} B g\right|_{Y} \leq \frac{C_{T}}{t^{\alpha}}|g|_{U},\left|e^{A t} G w\right|_{Y} \leq \frac{C_{T}}{t^{\alpha}}|w|_{V}, \\
& 0<\alpha<1,0<t \leq T
\end{aligned}
$$


Remark 3.1 We note that the singular estimate in (3.2) is automatically satisfied when the control operator is bounded $U \rightarrow Y$. Also, when semigroup $e^{A t}$ is analytic and the operator $B$ is $\alpha$ bounded (ie $R(\lambda, A)^{\alpha} B: U \rightarrow Y$ is bounded with $\alpha<1$, the singular estimate is just a consequence of analyticity . It will be shown later how one can arrive at such estimate. It is important to notice that our treatment does not assume a-priori analyticity of the resulting semigroup. In fact, in typical applications to fluid structure interactions, the semigroup is not analytic due to the presence of hyperbolic component associated with the dynamics of the wave equation modeling the elastic solid. However, the strongly damped waves $\rho>0$ lead to analytic semigroups that describe the model. This highly non-trivial fact will be shown later in Section 4.

The min-max game theory problem for the dynamics (3.1) satisfying (3.2) is as follows:

For any $y_{0} \in Y$, find a solution $\left\{y^{*}\left(t, y_{0}\right), g^{*}\left(t, y_{0}\right), w^{*}\left(t, y_{0}\right)\right\}$ on $(0, T)$, such that it solves the following problem:

$$
\sup _{w \in L_{2}(V)} \inf _{g \in L_{2}(U)} \int_{0}^{T}\left\{|R y(t)|_{Z}^{2}+|g(t)|_{U}^{2}-\gamma^{2}|w(t)|_{V}^{2}\right\} d t
$$

where $R$ is a linear bounded observation operator from $Y$ to another Hilbert space $Z$, and $\gamma$ is a positive constant.

Thus, qualitatively, the min-max game problem consists in finding the minimal cost of the system under the worst disturbance. For this problem, we obtain a critical value $\gamma_{c}$ for $\gamma$, and prove the existence and uniqueness of the solution under the condition $\gamma>\gamma_{c}$ for all initial conditions through a variational approach, while for $\gamma<\gamma_{c}$ there is no solution. The key result is that the solution pair (control $g^{*}$, disturbance $w^{*}$ ) can be expressed in pointwise feedback form of the dynamic $y^{*}$ :

$$
\begin{aligned}
& g^{*}\left(t, y_{0}\right)=-B^{*} P(t) y^{*}\left(t, y_{0}\right) ; w^{*}\left(t, y_{0}\right)=\gamma^{-2} \\
& G^{*} P(t) y^{*}\left(t, y_{0}\right) 0 \leq t \leq T
\end{aligned}
$$

where $P(t)$ satisfies an operator Riccati differential equation. Because the abstract system (3.1) is infinite dimensional with unbounded generator $A$, and highly unbounded control and disturbance operator $B$ and $G$, as noted before, the wellposedness of all quantities as well as of the associated Riccati differential equation is a fundamental technical issue (see Theorem 3.1). The presence of the "singular estimate" which substitutes for the lack of regularity of semigroup turns out (through a technical tour-de-force) to guarantee that the feedback operators $-B^{*} P(t)$ and $G^{*} P(t)$ are both bounded and, in fact, coincide with the sought after operators $K(t)$ and $D(t)$ in the formula (2.8).

Remark 3.2 The corresponding case, under singular estimates (3.2) for $T<\infty$ is studied in [27]. Instead, [28] studies the min-max problem in the abstract hyperbolic case for $T<\infty$.
The theory referred to above is based on the abstract model (3.1), and the result is optimal and complete in showing the validity for the construction of the associated non-standard Riccati equation. This allows one to include several physically relevent illustrations such as structural acoustic chambers, thermoelastic plates, composite beams, etc, for this abstract theory. However, the fluid-structure interaction model we are interested in happens to be more pathological. Its study requires solving additional technical and conceptual obstacles because such model fails the critical assumption of our original theory, the "singular estimates" (3.2) from the control/disturbance space to the state space $Y$. In fact, in the present case, a weaker "singular estimate" holds true, not in the original state space, but in a space slightly larger. This is due, intrinsically, to the fact that there is a mismatch between the regularity of the hyperbolic component and the regularity of the parabolic component at the interface $\Gamma_{s}$. As a consequence, the terms " $e^{A t} B u$ " and " $e^{A t} G w$ " no longer belong to the state space $Y$, and thus (3.2) fails in the present model. Our treatment in this part is to introduce a weaker (and more general) singular estimate condition, the Output Singular Estimate (the original singular estimate is then called the State Singular Estimate correspondingly). Then we extend our original theory to this new Output Singular Estimate PDE system.

\subsection{Abstract Min-Max Game Theorem for the Output Singular Estimate PDE System}

In this section, we develop the general min-max game theory in the context of abstract dynamics, with unbounded control and disturbance actions.

Abstract Dynamics. Let $U$ (control), $Y$ (the state), $Z$ (observation) and $V$ (disturbance) be given Hilbert spaces. We consider the dynamics governed by the state equation

$y_{t}=A y+B g+G w ;$ on $\left[\mathrm{D}\left(A^{*}\right)\right] ; y(0)=y_{0} \in Y$,

and subject to the following assumptions, to be maintained throughout the paper.

Hypotheses. Let $U$ (control), $Y$ (state), $Z$ (observation) and $V$ (disturbance) be given Hilbert space.

(H.1) $A: Y \supset \mathrm{D}(A) \rightarrow Y$ is the infinitesimal generator of a strongly continuous (s.c.) semigroup $e^{A t}$ on $Y, t \geq 0$.

(H.2) $B$ is a linear operator on $U=\mathrm{D}(B) \rightarrow\left[\mathrm{D}\left(A^{*}\right)\right]^{\prime}$, satisfying the condition $R(\lambda, A) B \in \mathrm{L}(U ; Y)$, for some $\lambda \in \rho(A)$, where $R(\lambda, A)$ is the resolvent of $A$ and $\rho(A)$ is the resolvent set of the $A$. (This assumption is automatically satisfied if $B \in \mathrm{L}(U, Y)$ i.e. $B$ is bounded as in the standard case)

(H.3) $G$ is a linear operator on $V=\mathrm{D}(G) \rightarrow\left[\mathrm{D}\left(A^{*}\right)\right]^{\prime}$, satisfying the condition $R(\lambda, A) G \in \mathrm{L}(V ; Y)$, for some $\lambda \in \rho(A)$. (This assumption is automatically satisfied if $G \in \mathrm{L}(V, Y)$ i.e. $G$ is bounded as in the standard case)

(H.4) Let $R: \mathrm{D}(R) \rightarrow Z$ be a linear operator, such that

$\mathrm{D}(R) \supset\left\{e^{A t} B U, 0<t \leq T\right\} \cup\left\{e^{A t} G V, 0<t \leq T\right\} \cup Y$ 
(H.5) The triple $\{A, B, R\}$ satisfies the following Output Singular Estimate Condition: There exists $0<\alpha<1$ and a constant $C_{T}>0$ such that

$$
\begin{aligned}
& \left|\operatorname{Re}^{A t} B\right|_{L(U ; Z)}=\left|B^{*} e^{A^{*} t} R^{*}\right|_{L(Z ; U)} \leq \frac{C_{T}}{t^{\alpha}}, 0<t \leq T \\
& \text { or } \operatorname{Re}^{A t} B g \in_{\alpha} C([0, T] ; Z), \quad \forall g \in U
\end{aligned}
$$

where $(B g, y)_{Y}=\left(g, B^{*} y\right)_{U}, g \in U, y \in \mathrm{D}\left(B^{*}\right) \supset \mathrm{D}\left(A^{*}\right)$. The function space ${ }_{\alpha} C([0, T] ; \cdot)$ is defined in () below.

(H.6) With $R$ as in (H.4), the triple $\{A, G, R\}$ satisfies the following Output Singular Estimate Condition:

$$
\begin{aligned}
& \left|R e^{A t} G\right|_{L(V ; Z)}=\left|G^{*} e^{A^{*} t} R^{*}\right|_{L(Z ; V)} \leq \frac{C_{T}}{t^{\alpha}}, 0<t \leq T \\
& \text { or } R e^{A t} G w \in_{\alpha} C([0, T] ; Z), \forall w \in V
\end{aligned}
$$

where $(G w, y)_{Y}=\left(w, G^{*} y\right)_{V}, w \in V$. For notational simplification, we take the same constant $\alpha$ in (5.3) and (5.4).

(H.7) With $R$ as in (H.4), $R \in \mathrm{L}(Y ; Z)$

Remark 3.3 If $B, G$ and $R$ are all bounded operators, then assumptions (H.2)-(H.7) are automatically satisfied. More siginificantly, we shall show that the above abstract assumptions (H.1)-(H.7) are a-fortiori satisfied in the case of fluid-structure interaction problem (2.1) with appropriately defined input-output spaces. Actually, assumption (H.6) accommodates also the case where the deterministic disturbance acts at the interface $\Gamma_{s}$. Thus, model (2.1) where however now both control $g$ and disturbance $w$ act on, say, two distinct portions of the interface $\Gamma_{s}$ is included in the present abstract setting. This is explicitly given at the end of Section 4.

Remark 3.4 Note that the Hypothesis (H.5) requires some balance between singularity of the control operator $B$ and the observation $R$. The observation is $R$ is allowed to mitigate strong singularity of $B$. Similar phenomenon applies to the disturbance $G$ and its relation to observation $R$ in the hypothesis (H.6) .

Notation For further reference, we next define the Banach space ${ }_{r} C([s, T] ; X)$, where $0<r<1$. This space measures the singularity on the left point $s$. The topology on ${ }_{r} C([s, T], X)$ is defined as $([21,26]$ p 3$)$ :

$$
\begin{aligned}
& { }_{r} C([s, T] ; X)=\{f \in C((s, T] ; X): \\
& \left.|f|_{r} C(s, T] ; X\right) \\
& \left.\equiv \sup _{s<t \leq T}(t-s)^{r}|f(t)|_{X}\right\}
\end{aligned}
$$

Min-max game theory problem over finite time interval $[0, T]$ For a fixed $0<T<\infty$ and fixed $\gamma>0$, we associate with (3.5) the cost functional

$$
\begin{aligned}
& J\left(g, w ; y_{0}\right) \equiv J\left(g, w ; y(g, w) ; y_{0}\right)= \\
& \int_{0}^{T}\left[|R y(t)|_{Z}^{2}+|g(t)|_{U}^{2}-\gamma^{2}|w(t)|_{V}^{2}\right] d t
\end{aligned}
$$

where $y(t)=y\left(t ; y_{0}\right)$ is the solution of (3.5) due to $g(t)$ and $w(t)$. What we are going to study is the following game theory problem

$$
\sup _{w \in L_{2}(V)} \inf _{g \in L_{2}(U)} J\left(g, w ; y_{0}\right)
$$

where the infimum is taken over all $g \in L_{2}(0, T ; U)$ for $w \in L_{2}(0, T ; V)$ fixed, and the supremum is then taken over all $w \in L_{2}(0, T ; V)$.

Abstract Theory Equipped with the above assumptions and properties, we have [24] the following result for the abstract dynamical model (3.5).

Theorem 3.1 Assume (H.1)-(H.7). Then there exists a critical value $\gamma_{c} \geq 0$, such that:

1. If $\gamma_{c}>0$ and $0<\gamma<\gamma_{c}$, then taking the supremum in $w$ leads to $+\infty$ for all initial conditions $y_{0} \in Y$; that is, there is no finite solution of the game theory problem (3.10)

2. If $\gamma>\gamma_{c}$, then: there exists a unique solution $\left\{g^{*}\left(\cdot ; y_{0}\right), w^{*}\left(\cdot ; y_{0}\right), y^{*}\left(\cdot ; y_{0}\right)\right\}$ of the game theory problem (3.10)

3. Moreover there exists a bounded non-negative selfadjoint operator $P(t)=P^{*}(t) \in \mathrm{L}(Y), \quad 0 \leq t \leq T, \quad$ defined explicitly in terms of the problem data, such that: $P(t)$ continuous : $Y \rightarrow C([0, T] ; Y)$

4. $\left|B^{*} P(t) x\right|_{U}+\left|G^{*} P(t) x\right|_{V} \leq C_{T}|x|_{Y}, 0 \leq t \leq T ; 0 \leq \alpha<1$

5. $P(t)$ defines the cost functional in (4.7b) of the solution of the min-max game problem initiating at the point $x \in Y$ and at the time $t$, over the interval $[t, T]$, for all $t \in[0, T]$ :

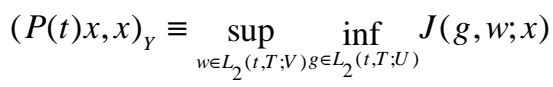

6. $P(t)$ satisfies a non-standard differential Riccati equation: for all $x, y \in \mathrm{D}(A)$, we have $P(T)=0$

$$
\left\{\begin{aligned}
(\dot{P}(t) x, y)_{Y}= & -(R x, R y)_{Y}-(P(t) x, A y)_{Y}-(P(t) A x, y)_{Y} \\
& +\left(B^{*} P(t) x, B^{*} P(t) y\right)_{U}-\gamma^{-2}\left(G^{*} P(t) x, G^{*} P(t) y\right)_{V}
\end{aligned}\right.
$$

7. The following pointwise feedback relations hold true for the min-max game theory solution:

$$
\begin{aligned}
& g^{*}(t ; x)=-B^{*} P(t) y^{*}(t ; x) \in C([0, T] ; U), x \in Y \\
& w^{*}(t ; x)=\gamma^{-2} G^{*} P(t) y^{*}(t ; x) \in C([0, T] ; V), x \in Y
\end{aligned}
$$

8. The operator $R \Phi(t, s) x \equiv R y^{*}(t, s ; x) \in C([s, T] ; Z), x \in Y$ is an evolution type operator, which is strongly continuous in each variable $t$ and $s$ separately, holding the other fixed. Moreover, for all $x \in \mathrm{D}(A), \Phi(t, s) x$ is differentiable with respect to $t$ in the dual sense on $\left[\mathrm{D}\left(A^{*}\right)\right]^{\prime}$; that is

$$
\frac{d}{d t} \Phi(t, s) x=\left[A-B B^{*} P(t)+\gamma^{-2} G G^{*} P(t)\right] \Phi(t, s) x \in C([s, T] ; Y) \text { (3.15) }
$$

9. $R \Phi(t, s) B$ and $R \Phi(t, s) G$ satisfy the same singular estimates as $R e^{A(t-s)} B$ and $R e^{A(t-s)} G$ in (H.5), (H.6); that is: 
$R \Phi(t, s) B g, R \Phi(t, s) G w \in \in_{\alpha} C([s, T] ; \cdot)$,

10. Finally, for all $x \in \mathrm{D}(A), R \Phi(t, s) x$ is differentiable with respect to $S$ in the space ${ }_{\alpha} C([s, t] ; Z)$, and

$\frac{d}{d s}[R \Phi(t, s) x]=-R \Phi(t, s)$

$\left[A-B B^{*} P(s)+\gamma^{-2} G G^{*} P(s)\right] x \in_{\alpha} C([s, t] ; Z)$

Remark 3.5 If B, G, R are bounded, Then Theorem 3.1 holds true under only one assumption (H.1).

Proof. Indeed, since the operator $B$ is bounded, then $R(\lambda, A) B \in \mathrm{L}(\mathrm{U}, Y)$, as a composition of two bounded operators. Thus (H.2) is satisfied automatically. The same applies to the disturbance $G$ in Hypothesis (H.3). When $R$ is bounded then $\mathrm{D}(R)$ coincides with the entire space, so that (H.4) is an empty assumptions. Singular estimate Hypotheses in (H.5) and (H.6) are automatically satisfied with $\alpha=0$. There is no singularity at the origin. Assumption (H.7) holds on the strength of strong continuity of the semigroup combined with the boundedness of $R$.

\section{ELASTIC AND VISCO-ELASTIC STRUCTURE GAME THEORY PROBLEM}

FLUID

Having at our disposal the abstract theory we are back to concrete application in the context of fluid-structure interaction.

\subsection{The Model}

The aim of this section is to put the original control problem within the abstract framework. For this we need to identify suitable operators describing the abstract setup.

Boundary Control/Boundary and Interior Disturbance model: In the present model, both controls $g_{0}$, $g_{1}$ and disturbance $w_{3}$ act at the interface between the two media. The disturbances $w_{1}$ and $w_{2}$ affect the fluid and the elastic body. To describe the model, we introduce the Cauchy Polya tensor discribing the fluid velocity and the pressure:

$\mathrm{T}(u, p) \equiv \varepsilon(u)-p I, \varepsilon(u) \equiv \frac{1}{2}\left(\nabla u+\nabla^{T} u\right)$

We shall also introduce functions localizing the effect of disturbance to some subregions of the specified domains. To this end, let $\phi_{\omega_{f}}(x)$ denotes a smooth function that is supported in a open set $\omega_{f} \subset \Omega_{f}$. Similarly $\varphi_{\omega_{s}}(x)$ will localize in a smooth manner to a subset $\omega_{s} \subset \Omega_{s}$. The PDE model under consideration is the following:

$$
\left\{\begin{array}{rrr}
u_{t}-\operatorname{div} \mathrm{T}(u, p)+L_{f} u=\varphi_{\omega_{f}} w_{1} & \text { in } & Q_{f} \equiv \Omega_{f} \times(0, T] \\
\operatorname{div} u=0 & \text { in } & Q_{f} \equiv \Omega_{f} \times(0, T] \\
v_{t t}-\operatorname{div} \sigma\left(v+\rho v_{t}\right)=\varphi_{\omega_{s}} w_{2} & \text { in } & Q_{s} \equiv \Omega_{s} \times(0, T] \\
v_{t}=u+\rho g_{0} & \text { in } & \Sigma_{s} \equiv \Gamma_{s} \times(0, T] \\
u=0 & \text { in } & \Sigma_{f} \equiv \Gamma_{f} \times(0, T] \\
\sigma\left(v+\rho v_{t}\right) \cdot v=\mathrm{T}(u, p) \cdot v-g_{1} & \text { in } & \Sigma_{s 1} \equiv \Gamma_{s 1} \times(0, T] \\
\sigma\left(v+\rho v_{t}\right) \cdot v=\mathrm{T}(u, p) \cdot v-w_{3} & \text { in } & \Sigma_{s 2} \equiv \Gamma_{s 2} \times(0, T]
\end{array}\right.
$$

with the initial data $u(0, \cdot)=u_{0}$ in $\Omega_{f}, v(0, \cdot)=v_{0}, v_{t}(0, \cdot)=v_{1}$ in $\Omega_{s}$ Here $\Gamma_{s 1} \cup \Gamma_{s 2}=\Gamma_{s}, \bar{\Gamma}_{s 1} \cap \bar{\Gamma}_{s 2}=\varnothing$. That is, the control $g$ and disturbance $w$ act on two distinct portions of the interface $\Gamma_{s}$. The linear operator $L_{f} u$ is a first order differential operator. In practical applications to Navier Stokes equation, this operator results from linearization of Navier Stokes operator and is given as in (2.5): where $y_{e}$ is an equilibrium point corresponding to a static but forced version of $\mathrm{N}-\mathrm{S}$ equation

$\operatorname{divT}\left(y_{e}, p\right)+\left(y_{e} \cdot \nabla\right) y_{e}=f, \operatorname{div} y_{e}=0, y_{e}=0$ on $\Gamma_{f}$

with some force $f \in L_{2}\left(\Omega_{f}\right)$. The force $f$ can also be seen as a fixed disturbance for the model.

\subsection{Specialization of Abstract Model to the Fluid- Structure Interaction Model}

In this section, we adapt the abstract theory we have developed to the fluid structure interaction model. The abstract spaces of Section 4 associated with the coupled PDE model (4.2) are given by:

$$
\begin{aligned}
& \mathrm{H} \equiv H \times\left(H^{1}\left(\Omega_{s}\right)\right)^{n} \times\left(L_{2}\left(\Omega_{s}\right)\right)^{n} \text { for }\left\{u, v, v_{t}\right\} \\
& \mathrm{U} \equiv\left(H^{1 / 2}\left(\Gamma_{s}\right)\right)^{n} \times\left(L_{2}\left(\Gamma_{s 1}\right)\right)^{n} \text { for } g=\left(g_{0}, g_{1}\right) \\
& \mathrm{V} \equiv\left(L_{2}\left(\Omega_{f}\right)\right)^{n} \times\left(L_{2}\left(\Omega_{s}\right)\right)^{n} \times\left(L_{2}\left(\Gamma_{s}\right)\right)^{n} \text { for } w=\left(w_{1}, w_{2}, w_{3}\right)
\end{aligned}
$$

where

$$
H \equiv\left\{u \in\left(L_{2}\left(\Omega_{f}\right)\right)^{n}: \operatorname{div} u=0,\left.u \cdot v\right|_{\Gamma_{f}}=0\right\}
$$

We moreover define the space

$$
E \equiv\left\{u \in\left(H^{1}\left(\Omega_{f}\right)\right)^{n}: \operatorname{div} u=0,\left.u\right|_{\Gamma_{f}}=0\right\}
$$

Notation: Henceforth, we shall drop the symbol $(\cdot)^{n}$, $n=2,3$ for all Sobolev spaces $H^{s}$ and $L_{2}$ spaces pertaining to $u$ and $v$, for the sake of simplicity of notation. Accordingly, say on the domain $\Omega_{s}$ and corresponding boundary $\Gamma_{s}$, the $L_{2}$-inner products are denoted by

$\left(v_{1}, v_{2}\right)_{s}=\int_{\Omega_{s}} v_{1} \cdot v_{2} d \Omega_{s} ;\left\langle u_{1}, u_{2}\right\rangle=\int_{\Gamma_{s}} u_{1} \cdot u_{2} d \Gamma_{s}$

On $\Omega_{f}$, we use $\left(u_{1}, u_{2}\right)_{f}=\int_{0} u_{1} u_{2} d \Omega_{f}$. Moreover the space $E$ is topologized with respect to the inner product

$$
\left(u_{1}, u_{2}\right)_{E}=\left(u_{1}, u_{2}\right)_{1, f} \equiv \int_{\Omega_{f}} \varepsilon\left(u_{1}\right) \cdot \varepsilon\left(u_{2}\right) d \Omega_{f}
$$

We also denote the induced norm by $|\cdot|_{1, \Omega_{f}}$ which is equivalent to the usual $H^{1}\left(\Omega_{f}\right)$ norm via Korn's inequality and Poincaré's inequality [29]:

$|u|_{1, \Omega_{f}}=\left(\int_{\Omega_{f}}|\varepsilon(u)|^{2} d \Omega_{f}\right)^{1 / 2}$ 
The space $H^{1}\left(\Omega_{s}\right)$ is topologized with respect to the inner product given by

$$
(v, z)_{1, \Omega_{s}} \equiv \int_{\Omega_{s}} v \cdot z d \Omega_{s}+\int_{\Omega_{s}} \sigma(v) \cdot \varepsilon(z) d \Omega_{s}
$$

so that the \|\|$_{1, \Omega_{s}}$ norm induced by the inner product above is

$$
|v|_{1, \Omega_{s}}^{2}=\int_{\Omega_{s}} \sigma(v) \cdot \varepsilon(v) d \Omega_{s}+|v|_{0, \Omega_{s}}^{2}
$$

This again equivalent to the usual $H^{1}\left(\Omega_{s}\right)$ norm by Korn's inequality [29].

Sobolev's norms of order $S$ are denoted by $|u|_{s, \Omega}=|u|_{H^{s}(\Omega)}$ ,$|u|_{s, \Gamma}=|u|_{H^{s}(\Gamma)}$. We shall also use the abbreviated notation $|u|=|u|_{0, \Omega}$

With $T>0$ preassigned, the quadratic cost functional corresponding to (4.2) is then

$$
\begin{aligned}
& J(u, v, g, w)=\int_{0}^{T}\left(|g(t)|_{U}^{2}+\left|R_{1} u(t)\right|_{L_{2}\left(\Omega_{f}\right)}^{2}+\right. \\
& \left.\left|R_{2} v(t)\right|_{H^{1}\left(\Omega_{s}\right)}^{2}+\left|R_{3} v_{t}(t)\right|_{L_{2}\left(\Omega_{s}\right)}^{2}\right) d t \\
& -\gamma^{2} \int_{0}^{T}|w(t)|_{V}^{2} d t
\end{aligned}
$$

where $g=\left(\rho g_{0}, g_{1}\right)$ and

$$
R_{1} \in \mathrm{L}\left(L_{2}\left(\Omega_{f}\right)\right), R_{2} \in \mathrm{L}\left(L_{2}\left(H^{1}\left(\Omega_{s}\right)\right)\right), R_{3} \in \mathrm{L}\left(L_{2}\left(\Omega_{s}\right)\right)
$$

The min-max game problem corresponding to (4.10) is the following:

$$
\sup _{w \in L_{2}(\mathrm{~V})} \inf _{g \in L_{2}(\cup)} J(u, v, g, w)
$$

where $u, v$ satisfy () with given $g, w$.

We next transform the PDE System (4.2) into the abstract state equation and then use the abstract theory in Section 3 to maxi-minimize the cost functional (4.10). The literature already contains two treatments leading to the abstract model corresponding to this PDE system. One approach introduced in [30] and pursued in [31, 32] - eliminates the pressure by introducing two suitable Green's maps of appropriate elliptic problems in $\Omega_{f}$; one with Dirichlet datum on $\Gamma_{s}$ and homogeneous Neumann datum on $\Gamma_{f}$; the second, the other way around: homogeneous Dirichlet datum in $\Gamma_{s}$ and Neumann datum on $\Gamma_{f}$. This approach was actually studied for the case of interest in these references, where $g \equiv 0$ in $\Gamma_{s}$. The second approach, instead, is variational: it was presented in [33-36]. We shall use this approach here. We first define the following operators: $A: E \rightarrow E^{\prime}$, such that

$$
(A u, \varphi)_{\Omega_{f}}=-(\mathrm{T}(u, p), \varepsilon(\varphi))_{\Omega_{f}}=-(\varepsilon(u), \varepsilon(\varphi))_{\Omega_{f}}, \varphi \in E
$$

$A$ is linear, bounded from $E$ to $E^{\prime}$ and coercive. This allows us to consider operator $A$ as acting on $\mathbb{H}$ with domain

$\mathrm{D}(A)=\left\{u \in E:\left|(\varepsilon(u), \varepsilon(\varphi))_{f}\right| \leq C|\varphi|_{E}\right\}$.

Thus, $A$ is negative, self-adjoint and generates an analytic semigroup $e^{A t}$ on $H$. In addition, we define the Neumann map $N: L_{2}\left(\Gamma_{s 1}\right) \times L_{2}\left(\Gamma_{s 2}\right) \rightarrow \mathrm{H}$, given by

$N\left(\begin{array}{c}\phi_{1} \\ \phi_{2}\end{array}\right)=\psi \Leftrightarrow\left\{\psi \in \mathrm{H}:(\varepsilon(\psi), \varepsilon(\varphi))_{\Omega_{f}}=\left\langle\phi_{1}, \varphi\right\rangle_{\Gamma_{s 1}}+\left\langle\phi_{2}, \varphi\right\rangle_{\Gamma_{s 2}}, \varphi\right.$

The Neumann map $N$ is linear bounded from $H^{-1 / 2}\left(\Gamma_{s_{1}}\right) \times H^{-1 / 2}\left(\Gamma_{s_{2}}\right)$ to $H^{1}\left(\Omega_{f}\right)[33,35]$. We are in a position to define the dynamics of the overall coupled system. Let

$\mathrm{A}=\left(\begin{array}{ccc}A-L_{f} & A N \sigma() \cdot v & \rho A N \sigma() \cdot v \\ 0 & 0 & I \\ 0 & \operatorname{div} \sigma() & \rho \operatorname{div} \sigma()\end{array}\right)$

By [36], because $L_{f}$ is relatively bounded (by Lemma 5.1) from $\mathrm{D}(A)$ to $\mathrm{H}$, the pertubation $A-L_{f}$ still generates an analytic semigroup. It was shown in [33]. A with domain $\mathrm{D}(\mathrm{A}) \subset \mathrm{H} \rightarrow \mathrm{H}$ defined as follows

$\mathrm{D}(\mathrm{A})=\left\{(u, v, z) \in \mathrm{H}: u \in E, A(u+N \sigma(v+\rho z) \cdot v) \in \mathrm{H}, z \in H^{1}\left(\Omega_{s}\right)\right.$,

$\operatorname{div} \sigma(v+\rho z) \in L_{2}\left(\Omega_{s}\right),\left.z\right|_{\Gamma_{s}}=\left.u\right|_{\Gamma_{s}}$ in $\left.H^{1 / 2}\left(\Gamma_{s}\right)\right\}$

will generate a strongly continuous semigroup $e^{\mathrm{A} t}$ on $H$. This semigroup will be shown analytic when $\rho>0$.

\subsection{Main Result-Min-Max Games for Fluid-Structure Interaction Model}

In what follows we shall refer to: the generator $\mathrm{A}$ given by (4.15), control operator B in (5.40) and disturbance operator $\mathrm{G}$ given by (5.37) phase space $\mathrm{H}=\mathrm{H} \times H^{1}\left(\Omega_{s}\right) \times L_{2}\left(\Omega_{s}\right)$, control space $\mathrm{V}=H^{1 / 2}\left(\Gamma_{s}\right) \times L_{2}\left(\Gamma_{s}\right)$, disturbance space $\mathrm{V}=L_{2}\left(\Omega_{f}\right) \times L_{2}\left(\Omega_{s}\right) \times L_{2}\left(\Gamma_{s_{2}}\right)$. The adjoints of $\mathrm{B}: \mathrm{U} \rightarrow \mathrm{H}$ and $\mathrm{G}: \mathrm{V} \rightarrow H$ are given by

$\mathrm{B}^{*}(u, v, z)=\left[\left.u\right|_{\Gamma_{s}}, \rho \Lambda_{\tau}^{-1} \sigma(z) \cdot v\right]$ for $(u, v, z) \in D\left(\mathrm{~A}^{*}\right)$ where $\Lambda_{\tau}$ is a positive first order tangential operator on $\Gamma_{s}$ : $\left\|\Lambda_{\tau}^{1 / 2} u\right\|_{L_{2}\left(\Gamma_{s}\right)}=\|u\|_{H^{1 / 2}\left(\Gamma_{s}\right)}$

and

$\mathrm{G}^{*}(u, v, z)=\left[\varphi_{\omega_{f}} u, \varphi_{\omega_{s}} z,\left.u\right|_{\Gamma_{s_{2}}}\right]$.

Theorem 4.1 Let's assume that the static disturbance $f$ is in a bounded set in $L_{2}(\Omega)$. In reference to model (4.2) with $\rho>0$ and the min-max game problem (4.11), there 
exists a critical $\gamma_{c}>0$, for each initial condition in $\mathcal{H}$, that is, $y_{0}=\left(u_{0}, v_{0}, v_{1}\right) \in \mathrm{H} \times H^{1}\left(\Omega_{s}\right) \times L_{2}\left(\Omega_{s}\right)=\mathrm{H}$,

1. Nonexistence. if $0<\gamma<\gamma_{c}, J(u, v, g, w) \rightarrow \infty$ as we take supremum over $w=\left(w_{1}, w_{2}, w_{3}\right) \in L_{2}(\mathrm{~V})$. Thus there is not finite solution for (4.11) for any initial condition $y_{0} \in \mathcal{H}$.

2. Existence. if $\gamma>\gamma_{c}$, then for each initial condition $y_{0} \in \mathcal{H}$ there exists a unique control $g^{*}=\left(g_{0}^{*}, g_{1}^{*}\right) \in C([0, T]$, $\left.H^{1 / 2}\left(\Gamma_{s}\right) \times L_{2}\left(\Gamma_{s}\right)\right)$, a unique disturbance $\left(w_{1}^{*}, w_{2}{ }^{*}, w_{3}^{*}\right) \in C([0, T]$, $\left.\left.L_{2}\left(\Omega_{f}\right) \times L_{2}\left(\Omega_{s}\right)\right) \times L_{2}\left(\Gamma_{s}\right)\right)$ and the corresponding optimal state $y^{*}(t)=\left(u^{*}(t), v^{*}(t), v_{t}^{*}(t)\right) \in C\left([0, T], \mathrm{H} \times H^{1}\left(\Omega_{s}\right) \times L_{2}\left(\Omega_{s}\right)\right)$

such that

$$
J\left(y^{*}, g^{*}, w^{*}\right)=\sup _{w \in L_{2}(\mathrm{~V})} \inf _{g \in L_{2}(U)} J(y(g, w), g, w)
$$

Furthermore, for all $t \in[0, T]$

(a) $\left|g_{1}^{*}(t)\right|_{\left(L_{2}\left(\Gamma_{s}\right)\right.}+\rho\left|g_{0}^{*}(t)\right|_{H^{1 / 2}\left(\Gamma_{s}\right)} \leq C\left|y_{0}\right|_{H}$,

(b) $\left|w_{1}^{*}(t)\right|_{L_{2}\left(\Omega_{f}\right)}+\left|w_{2}^{*}(t)\right|_{L_{2}\left(\Omega_{s}\right)}+\left|w_{3}^{*}(t)\right|_{L_{2}\left(\Gamma_{s_{2}}\right)} \leq C\left|y_{0}\right|_{H}$.

$$
\left|v^{*}(t)\right|_{H^{1}\left(\Omega_{s}\right)}^{2}+\left|v_{t}^{*}(t)\right|_{L_{2}\left(\Omega_{s}\right)}^{2}+\left|u^{*}(t)\right|_{H}^{2} \leq C\left|y_{0}\right|_{H} .
$$

(c) Feedback Synthesis. There exists a positive selfadjoint $n \times n$ operator matrix $P(t)$ on $\mathrm{H}$, let $P(t)\left(v^{*}(t), v_{t}^{*}(t), u^{*}(t)\right)=\left(p_{1}(t), p_{2}(t), p_{3}(t)\right)$ with $p_{1}(t), \quad p_{2}(t)$ and $p_{3}(t)$ being $n$-dimensional vector function, such that the control is given by

$$
\begin{aligned}
& \left.g^{*}(t)=-\mathrm{B}^{*} P(t) y^{*}(t)=-\left.\left(p_{1}(t)\right)\right|_{\Gamma_{s}}, \rho \Lambda_{\tau}^{-1} \sigma\left(p_{3}(t)\right) \cdot v\right) \\
& w^{*}(t)=-\gamma^{-2} \mathrm{G}^{*} P(t) y^{*}(t)=-\gamma^{-2}\left[\varphi_{\omega_{f}} p_{1}(t), \varphi_{\omega_{s}} p_{3}(t),\left.p_{1}(t)\right|_{\Gamma_{s_{2}}}\right]
\end{aligned}
$$

(d) Boundedness of the Gains. The feedback operator $\mathrm{B}^{*} P(t) \in \mathrm{L}(\mathrm{H}, \mathrm{U})$ for all $0 \leq t \leq T$ with the estimate

$\left|\mathrm{B}^{*} P(t) y\right|_{\mathrm{U}} \leq C|y|_{\mathrm{H}}, \quad\left|\mathrm{G}^{*} P(t) y\right|_{L_{2}(\mathrm{v})} \leq C|y|_{\mathrm{H}}$

(e) Riccati Equation. $P(t)$ is the unique solution to the following non-standard Riccati equation: $P(T)=0$ and

$$
\left\{\begin{array}{l}
(\dot{P}(t) x, y)_{\mathrm{H}}=-(\mathrm{R} x, \mathrm{R} y)_{\mathrm{H}}-(P(t) x, \mathrm{~A} y)_{\mathrm{H}}-(P(t) \mathrm{A} x, y)_{\mathrm{H}}+ \\
\left(\mathrm{B}^{*} P(t) x, \mathrm{~B}^{*} P(t) y\right)_{\mathrm{U}}-\gamma^{-2}\left(\mathrm{G}^{*} P(t) x, \mathrm{G}^{*} P(t) y\right)_{\mathrm{V}}
\end{array}\right\}
$$

where $\quad \mathrm{R}=\left(R_{1}, R_{2}, R_{3}\right) \quad$ with $R_{1} \in \mathrm{L}\left(L_{2}\left(\Omega_{f}\right)\right), \quad R_{2} \in \mathrm{L}\left(H^{1}\left(\Omega_{s}\right)\right)$ , $R_{3} \in \mathrm{L}\left(L_{2}\left(\Omega_{s}\right)\right)$.

Remark 4.1 The main novelty and interest in Theorem 4.1 is the fact that the control action is unbounded (ie B is unbounded), yet we obtain fully satisfactory wellposedness of Riccati equation along with feedback synthesis that is point-wisely in time defined via gain operators that are bounded. Indeed $K(t) \equiv-\mathrm{B}^{*} P(t), D(t) \equiv \gamma^{2} \mathrm{G}^{*} P(t)$. This exhibits certain smoothing mechanism displayed by solutions of Riccati equations. The latter reflects the effects of singular estimate.

Remark 4.2 The theory presented above with $\rho>0$ does not require any smoothing (with respect to the state space) property imposed on the observation operator R. In fact, one can also consider unbounded observation (eg first order differential operator in the Neumann case). This is in contrast with other treatments where such smoothing was required $[25,35,36]$. When $\rho=0$, in which case only $g_{1}$ is active, the same result is valid with one difference that the observation should satisfy incremental smoothing property $\mathrm{R}=\left(R_{1}, R_{2}, R_{3}\right)$ with $R_{1} \in \mathrm{L}\left(L_{2}\left(\Omega_{f}\right)\right), R_{2} \in \mathrm{L}\left(H^{1-\varepsilon}\left(\Omega_{s}\right) \rightarrow H^{1}\left(\Omega_{s}\right)\right)$.

\section{PROOF OF THEOREM 4.1}

\subsection{Preliminaries}

The following Lemma is critical for the development.

Lemma 5.1 The operator $L_{f} u$ is a first order differential operator in $u$ bounded on $L_{2}\left(\Omega_{f}\right)$. This is to say

$\left|L_{f} u\right|_{L_{2}\left(\Omega_{f}\right)} \leq C|f|_{L_{2}\left(\Omega_{f}\right)}|\nabla u|_{L_{2}\left(\Omega_{f}\right)}$

Proof. By elliptic regularity [18], one has

$y_{e} \in H^{2}\left(\Omega_{f}\right) \cap H_{0}^{1}\left(\Omega_{f}\right), \operatorname{div} y_{e}=0$

and the following estimate is valid:

$\left|y_{e}\right|_{H^{2}\left(\Omega_{f}\right)} \leq C\left(|f|_{L_{2}\left(\Omega_{f}\right)}\right)$

By Sobolev's embeddings $(n<3)$

$\left|y_{e}\right|_{L_{\infty}\left(\Omega_{f}\right)} \leq C\left|y_{e}\right|_{H^{2}\left(\Omega_{f}\right)} \leq C\left(|f|_{L_{2}\left(\Omega_{f}\right)}\right)$

This gives

$\left|y_{e} \cdot \nabla u\right|_{L_{2}\left(\Omega_{f}\right)} \leq C\left(|f|_{L_{2}\left(\Omega_{f}\right)}\right)|\nabla u|_{L_{2}\left(\Omega_{f}\right)}$

As for the second term $u \cdot y_{e}$ in $L_{f} u$, we estimate as follows

$\left|u \cdot \nabla y_{e}\right|_{L_{2}\left(\Omega_{f}\right)} \leq|u|_{L_{6}\left(\Omega_{f}\right)}\left|\nabla y_{e}\right|_{L_{3}\left(\Omega_{f}\right)}$

Since $\nabla y_{e} \in H^{1}\left(\Omega_{f}\right)$ and $H^{1}\left(\Omega_{f}\right) \subset L_{6}\left(\Omega_{f}\right)$, we infer $\nabla y_{e} \in L_{6}\left(\Omega_{f}\right)$. This gives

$\left|u \cdot \nabla y_{e}\right|_{L_{2}\left(\Omega_{f}\right)} \leq C|u|_{H^{1}\left(\Omega_{f}\right)}\left|\nabla y_{e}\right|_{L_{6}\left(\Omega_{f}\right)}$

$\leq C|u|_{H^{1}\left(\Omega_{f}\right)}\left|y_{e}\right|_{H^{2}\left(\Omega_{f}\right)} \leq|u|_{H^{1}\left(\Omega_{f}\right)} C\left(|f|_{L_{2}\left(\Omega_{f}\right)}\right)$

And the final conclusion results from Poincare inequality.

\subsection{Generation of the Semigroup}

In what follows $y=\left(u, v, v_{t}\right)=(u, v, z) \quad$ and $y_{0}=\left(u_{0}, v_{0}, z_{0}\right)$. 
Theorem 5.1 The operator A generates a strongly continuous semigroup on $\mathrm{H}$ for all $\rho \geq 0$. When $L_{f}=0$ the corresponding semigroup is contractive.

Proof. In the case when $\rho=0$ the proof of this result is given in [33]. Similar argument can be applied also to the case $\rho>0$. To this end it suffice to note the following relation

$$
\begin{aligned}
& \operatorname{Re}(\mathrm{A} y, y)_{\mathrm{H}}=-\rho(\sigma(z), \varepsilon(z))_{s}-(\varepsilon(u), \\
& \varepsilon(u))_{f}+\left(L_{f} u, u\right), \quad y \in \mathrm{D}(\mathrm{A})
\end{aligned}
$$

The above equality is obtained by integration by parts with the use of cancellations occurring in the boundary conditions. By Korn's inequality one has

$$
(\sigma(z), \varepsilon(z))_{s} \geq \alpha|z|_{1, \Omega_{s}}^{2}
$$

This along with the $H^{1}$ boundedness of $L_{f} u$-see Lemma 5.1 - implies

$$
-\operatorname{Re}(\mathrm{A} y, y)_{H} \geq c\left[|u|_{1, \Omega_{f}}^{2}+\rho|z|_{1, \Omega_{s}}^{2}\right]-c_{f}|u|_{f}^{2}
$$

where $\quad c_{f}=0$ when $L_{f}=0$. The relation in (5.10) proves dissipativity of A. The proof of maximality follows now the same arguments as in [30,33].

In the visco-elastic case, when $\rho>0$ a much stronger conclusion holds.

Theorem 2.2 For $\rho>0$ the operator A generates an analytic semigroup of contractions on $\mathcal{H}$.

Proof. Here the argument is different and it boils down to obtaining suitable estimate for the resolvent operator. Our aim is to prove that there exist constants $C>0$ and $\omega>0$ such that

$$
|R(\lambda, \mathrm{A})|_{L(\mathrm{H})} \leq \frac{C}{|\lambda|}, \forall \operatorname{Re} \lambda>\omega
$$

To proceed with the estimate, we introduce the following change of variables.

$\zeta \equiv v+\rho v_{t}$

Then (see (5.2) with $g=0, w=0$ )

$\zeta_{t}=v_{t}+\rho v_{t t}=\rho^{-1}(\zeta-v)+\rho[\operatorname{div} \sigma(\zeta)]$

With the above notation we consider the following PDE system in the variables $(u, v, \zeta, p)$ :

$$
\left\{\begin{array}{l}
u_{t}-\operatorname{div} \mathrm{T}(u, p)-L_{f} u=0, \operatorname{div} u=0 \\
v_{t}+\rho^{-1} v=\rho^{-1} \zeta \\
\zeta_{t}-\rho \operatorname{div} \sigma(\zeta)=\rho^{-1}(\zeta-v)
\end{array}\right.
$$

accompanied by the boundary conditions on the interface

$$
\left\{\begin{array}{l}
\sigma(\zeta) \cdot v=\mathrm{T}(u, p) \cdot v, \text { on } \Sigma_{s} \\
\zeta=v+\rho u, \text { on } \Sigma_{s}, u=0 \text { on } \Sigma_{s}
\end{array}\right.
$$

This amounts, after elimination of the pressure, to the consideration of the following matrix operator

$\mathrm{A}_{1}=\left(\begin{array}{lll}A-L_{f} & 0 & A N \sigma() \cdot v \\ 0 & -\rho^{-1} I & \rho^{-1} I \\ 0 & -\rho^{-1} I & \rho \operatorname{div} \sigma()+\rho^{-1} I\end{array}\right)$

$\mathrm{D}\left(\mathrm{A}_{1}\right)=\left\{(u, v, \zeta) \in \mathrm{H}: u \in E, A(u+N \sigma(\zeta) \cdot v) \in \mathrm{H}, \zeta \in H^{1}\left(\Omega_{s}\right)\right.$,

$\operatorname{div} \sigma(\zeta) \in L_{2}\left(\Omega_{s}\right),\left.\zeta\right|_{\Gamma_{s}}=\left.[v+\rho u]\right|_{\Gamma_{s}}$ in $\left.H^{1 / 2}\left(\Gamma_{s}\right)\right\}$

We are looking for a solution $(u, v, \zeta) \in \mathrm{H}_{1} \equiv \mathrm{H} \times H^{1}\left(\Omega_{s}\right) \times L_{2}\left(\Omega_{s}\right)$ which satisfies

$\lambda(u, v, \zeta)=\mathrm{A}_{1}(u, v, \zeta)$

The above topological setup in $\mathcal{H}_{1}$ is equivalent to the original space $\mathcal{H}$ with the variables $\left(u, v, v_{t}\right)$. We note that (5.14) is a first order system of three equations (after elimination of the pressure) in the three variables. The first and the third equation are "parabolic" like and the second one is an abstract ODE. Parabolicity of equations involved is a good predictor for analytic behavior of the associated semigroup. However, the boundary conditions provide for substantial and unbounded coupling -thus potentially destroying analyticity of the uncoupled system. Our main effort goes into showing that these boundary traces can be handled and eventually lead to an overall analytic dynamics generated by $\mathrm{A}$. We note that the resolvent estimate for $\mathrm{A}$ is equivalent to that for $\mathrm{A}_{1}$ (when $\rho>0$ )

We shall estimate the resolvent operator corresponding to the system (5.14). This amounts to solving $(u, v, \zeta)=R\left(\lambda, \mathrm{A}_{1}\right)\left(h_{1}, h_{2}, h_{3}\right)$ where

$$
\left\{\begin{array}{l}
\lambda u-\operatorname{div} T(u, p)-L_{f} u=h_{1}, \operatorname{div} u=0 \\
\lambda v+\rho^{-1} v-\rho^{-1} \zeta=h_{2} \\
\lambda \zeta-\rho \operatorname{div} \sigma(\zeta)-\rho^{-1}(\zeta-v)=h_{3}
\end{array}\right.
$$

Where we take

$$
\left(h_{1}, h_{2}, h_{3}\right) \in \mathrm{H}_{1}=\mathrm{H} \times H^{1}\left(\Omega_{s}\right) \times L_{2}\left(\Omega_{s}\right)
$$

and the boundary conditions are the same as in (5.15). Adopting the notation from the previous section and neglecting the lower order terms, the latter have no impact on analyticity, we are left to consider the following system

$$
\left\{\begin{array}{l}
\lambda u-A u+A N(\sigma(\zeta) \cdot v)=h_{1} \\
\lambda v+\rho^{-1} v-\rho^{-1} \zeta=h_{2} \\
\lambda \zeta-\rho \operatorname{div} \sigma(\zeta)=h_{3} \\
\zeta=v+\rho u, \text { on } \Gamma_{s}
\end{array}\right.
$$

Multiplying the first equation by $u$ and the third by $\zeta$, integrating by parts and recalling $N^{*} A u=\left.u\right|_{\Gamma}$ yields

$$
\lambda|u|_{f}^{2}+|\nabla u|_{f}^{2}+\langle\sigma(\zeta) \cdot v, u\rangle=\left(h_{1}, u\right)_{f}
$$




$$
\lambda|\zeta|_{s}^{2}+\rho(\sigma(\zeta), \varepsilon(\zeta))_{s}-\rho\langle\sigma(\zeta) \cdot v, \zeta\rangle=\left(h_{3}, \zeta\right)_{s}
$$

Using boundary conditions $\zeta=v+\rho u$ on $\Gamma_{s}$,

$\rho \lambda|u|_{f}^{2}+\rho|\nabla u|_{f}^{2}+\lambda|\zeta|_{s}^{2}+\rho(\sigma(\zeta), \varepsilon(\zeta))_{s}+$

$\langle\sigma(\zeta) \cdot v, v\rangle=\rho\left(h_{1}, u\right)_{f}+\left(h_{3}, \zeta\right)_{s}$

The second equation in (5.20) is treated via $H^{1}$ inner product. This gives

$\operatorname{Re} \lambda|\nabla v|_{s}^{2}+\rho^{-1}|\nabla v|_{s}^{2}=\rho^{-1}(\nabla \zeta, \nabla v)_{s}+\left(\nabla h_{2}, \nabla v\right)_{s}$

$\leq \frac{\operatorname{Re} \lambda}{2}|\nabla v|_{s}^{2}+\frac{C_{\rho}}{\operatorname{Re} \lambda}|\nabla \zeta|_{s}^{2}+C \mid\left(\nabla v, \nabla h_{2}\right)_{s}$

(5.24) gives a very useful estimate reflecting upon the fact that $v$ is an ODE:

$\operatorname{Re} \lambda|\nabla v|_{s}^{2} \leq \frac{C}{\operatorname{Re} \lambda}|\nabla \zeta|_{s}^{2}+C\left|\left(\nabla v, \nabla h_{2}\right)_{s}\right|$

In order to eliminate boundary terms in the equation (5.23), we multiply $\zeta$ equation by $v$ and integrate by parts

$$
\lambda(\zeta, v)_{s}+\rho(\sigma(\zeta), \varepsilon(v))_{s}-\rho\langle\sigma(\zeta) \cdot v, v\rangle=\left(h_{3}, v\right)_{s}
$$

which gives (upon using (5.20),

$$
\begin{aligned}
& \langle\sigma(\zeta) \cdot v, v\rangle=\frac{\lambda}{\rho}(\zeta, v)_{s}+(\sigma(\zeta), \varepsilon(v))_{s}-\frac{1}{\rho}\left(h_{3}, v\right)_{s} \\
& =\frac{1}{\rho}\left(\rho^{-1}(\zeta-v)+h_{2}, \zeta\right)_{s}+(\sigma(\zeta), \varepsilon(v))_{s}-\frac{1}{\rho}\left(h_{3}, v\right)_{s} \\
& \leq C_{\rho}\left[\left|(\nabla \zeta, \nabla v)_{s}\right|+|\zeta|_{s}^{2}+|v|_{s}^{2}+\left|\left(h_{2}, \zeta\right)_{s}\right|+\left|\left(h_{3}, v\right)_{s}\right|\right]
\end{aligned}
$$

By Young's inequality

$(\nabla \zeta, \nabla v)_{s} \leq \varepsilon|\nabla \zeta|_{s}^{2}+C_{\varepsilon}|\nabla v|_{s}^{2}$

On the other hand, the second equation in (5.20) for $v$ with (5.24) gives along with (5.25)

$$
|\nabla v|_{s}^{2} \leq \frac{C}{(\operatorname{Re} \lambda)}\left(\left|\left(\nabla v, \nabla h_{2}\right)_{s}\right|+|\nabla \zeta|_{s}|\nabla v|_{s}\right)
$$

Hence,

$$
\begin{aligned}
& (\nabla \zeta, \nabla v)_{s} \leq \varepsilon|\zeta|_{1, \Omega_{s}}^{2}+\frac{C_{\varepsilon}}{\operatorname{Re} \lambda}\left(\left|\left(\nabla v, \nabla h_{2}\right)_{s}\right|+|\nabla \zeta|_{s}|\nabla v|_{s}\right) \\
& \leq\left(\varepsilon+C_{\varepsilon}(\operatorname{Re} \lambda)^{-1}\right)|\zeta|_{1, \Omega_{s}}^{2}+C_{\varepsilon}(\operatorname{Re} \lambda)^{-1} \\
& |v|_{1, \Omega_{s}}^{2}+\frac{C}{\operatorname{Re} \lambda}\left|\left(\nabla v, h_{2}\right)_{s}\right|
\end{aligned}
$$

After taking $\operatorname{Re} \lambda$ sufficiently large which is scaled to $\varepsilon^{-2}, \operatorname{Re} \lambda>c \varepsilon^{-2}$.

$(\nabla \zeta, \nabla v)_{s} \leq \varepsilon\left[|\zeta|_{1, \Omega_{s}}^{2}+|v|_{1, \Omega_{s}}^{2}\right]+C_{\varepsilon}\left|\left(\nabla v, h_{2}\right)_{s}\right|$

This gives, by using (5.27) $|\langle\sigma(\zeta) \cdot v, v\rangle| \leq \varepsilon\left[|\zeta|_{1, \Omega_{s}}^{2}+|v|_{1, \Omega_{s}}^{2}\right.$

$+C\left[|\zeta|_{s}^{2}+|v|_{s}^{2}+\left|\left(\nabla v, \nabla h_{2}\right)_{s}\right|+\left|\left(v, h_{3}\right)_{s}\right|+\left|\left(\zeta, h_{2}\right)_{s}\right|\right]$

The above estimates along with (5.23) lead to

$|u|_{1, \Omega_{f}}^{2}+\rho|\zeta|_{1, \Omega_{s}}^{2}+\operatorname{Re} \lambda \mid\left[|u|_{f}^{2}+|\zeta|_{s}^{2}\right]$

$\leq C_{\rho}\left[|v|_{s}^{2}+|\zeta|_{s}^{2}+\left|\left(u, h_{1}\right)_{f}\right|+\right.$

$\left.\left|\left(\nabla v, \nabla h_{2}\right)_{s}\right|+\left|\left(\zeta, h_{2}\right)_{s}\right|+\left|\left(v, h_{3}\right)_{s}\right|+\left|\left(\zeta, h_{3}\right)_{s}\right|\right]$

Taking $\operatorname{Re} \lambda$ large enough, recalling (5.29) along with the second equation in (5.20) yields to

$|u|_{1, \Omega}^{2}+|\zeta|_{1, \Omega_{s}}^{2}+|v|_{1, \Omega}^{2}$

$\leq C_{\rho}\left[\left|\left(u, h_{1}\right)_{f}\right|+\left|\left(\nabla v, \nabla h_{2}\right)_{s}\right|+\mid\left(\zeta, h_{2}\right)_{s}\right.$

$\left.|+|\left(v, h_{3}\right)_{s}|+|\left(\zeta, h_{3}\right)_{s} \mid\right]$

where the estimate is uniform for $|\lambda|$ large with $\operatorname{Re} \lambda>\omega>0$.

Recall that

$\mathrm{D}\left(\mathrm{A}^{1 / 2}\right)=\left\{(u, v, z) \in \mathrm{H}, u \in E, v \in H^{1}\left(\Omega_{s}\right), z \in H^{1}\left(\Omega_{s}\right), u=z\right.$ on $\left.\Gamma_{s}\right\}$.

Hence $u \in H^{1}\left(\Omega_{f}\right), v \in H^{1}\left(\Omega_{s}\right), \quad \zeta \in H^{1}\left(\Omega_{s}\right)$ and such that $\zeta=v+\rho u$, on $\Gamma_{s}$ is equivalent to the membership of $(u, v, \zeta) \in \mathrm{D}\left(\mathrm{A}_{1}^{1 / 2}\right)$. In view of this, (5.34) can be written as

$\left|\mathrm{A}_{1}^{1 / 2}(u, v, \zeta)\right|_{H_{1}}^{2} \leq C_{\rho}\left[\left|\left(u, h_{1}\right)_{f}\right|+\left|\left(\nabla v, \nabla h_{2}\right)_{s}\right|+\right.$

$\left.\left|\left(\zeta, h_{2}\right)_{s}\right|+\left|\left(v, h_{3}\right)_{s}\right|+\left|\left(\zeta, h_{3}\right)_{s}\right|\right]$

Thus, by duality, the solution to the resolvent equation $(u, v, \zeta)=R\left(\lambda, \mathrm{A}_{1}\right)\left(h_{1}, h_{2}, h_{3}\right)$ satisfies

$\left|\mathrm{A}_{1}^{1 / 2}(u, v, \zeta)\right|_{\mathrm{H}_{1}} \leq C\left|\mathrm{~A}_{1}^{-1 / 2}\left(h_{1}, h_{2}, h_{3}\right)\right|_{\mathrm{H}_{1}}$

or

$\left|\mathrm{A}^{1 / 2} R(\lambda, \mathrm{A}) F\right|_{\mathrm{H}} \leq C\left|\mathrm{~A}^{-1 / 2} F\right|_{\mathrm{H}}$

or equivalently to

$|\mathrm{A} R(\lambda, \mathrm{A}) F|_{\mathrm{H}} \leq C|F|_{H}, \operatorname{Re} \lambda>\omega$

Where the estimate is uniform with respect to $\lambda$ such that $\operatorname{Re} \omega>0$. The above, via the resolvent identity, proves (5.11), hence the the analyticity of the semigroup.

\subsection{Control Problem}

\subsubsection{Setting}

We introduce the abstract control and disturbance operators. First, when $g_{0}=0$ the control (Neumann) operator and the disturbance operator have the form 


$$
\mathrm{B}=\left(\begin{array}{c}
A N_{1} \\
0 \\
0
\end{array}\right), \mathrm{G}=\left(\begin{array}{ccc}
P \varphi_{\omega_{f}} & 0 & A N_{2} \\
0 & 0 & 0 \\
0 & \varphi_{\omega_{s}} & 0
\end{array}\right)
$$

Here $N_{1}(g)=N(g, 0), N_{2}(g)=N(0, g)$. When $g_{0} \neq 0$ (Dirichlet) it is convenient to provide a different representation for the operator A. Our aim is to incorporate Dirichlet action into the structure of the operator. Indeed, let $D: H^{s}\left(\Gamma_{s}\right) \rightarrow H^{s+1 / 2}\left(\Omega_{s}\right)$ denotes the usual harmonic (Dirichlet) extension of the boundary data into the interior. We shall denote by $A_{D}$ the corresponding elastic operator $\operatorname{div} \sigma$ with the zero Dirichlet data. The trace operator $\gamma_{0}$ denotes the restriction to the boundary: $\left.\gamma_{0} v \equiv v\right|_{\Gamma_{s}}$. Then the operator $\mathrm{A}$ has an equivalent representation

$$
\mathrm{A}=\left(\begin{array}{ccc}
A-L_{f} & A N \sigma() \cdot v & \rho A N \sigma() \cdot v \\
0 & 0 & I \\
-\rho A_{D} D \gamma_{0} & A_{D}-A_{D} D \gamma_{0} & \rho A_{D}
\end{array}\right)
$$

$\mathrm{D}(\mathrm{A})=\{(u, v, z) \in \mathrm{H}: u \in E, A(u+N \sigma(v+\rho z) \cdot v) \in \mathrm{H}$,

$$
\left.z \in H^{1}\left(\Omega_{s}\right), \operatorname{div} \sigma(v+\rho z) \in L_{2}\left(\Omega_{s}\right),\left.z\right|_{\Gamma_{s}}=\left.u\right|_{\Gamma_{s}} \text { in } H^{1 / 2}\left(\Gamma_{s}\right)\right\}
$$

and the operator $\mathrm{B}$ is given by

$\mathrm{B}\left(g_{0}, g_{1}\right)=\left(\begin{array}{c}A N_{1} g_{1} \\ 0 \\ \rho A_{D} D g_{0}\end{array}\right)$

In order to justify a new structure of A, it suffices to recall (4.15), definition of Dirichlet map and write

$\operatorname{div} \sigma(v)=\operatorname{div} \sigma(v)-\operatorname{div} \sigma\left(D \gamma_{0} v\right)=\operatorname{div} \sigma\left(v-D \gamma_{0} v\right)=A_{D}\left(v-D \gamma_{0} v\right)$

Similarly, after using compatibility conditions on the boundary $z=u$ on $\Gamma_{s}$ for $(u, v, z) \in \mathrm{D}(\mathrm{A})$

$\operatorname{div} \sigma(z)=\operatorname{div} \sigma(z)-\operatorname{div} \sigma\left(D \gamma_{0} u\right)=\operatorname{div} \sigma\left(z-D \gamma_{0} u\right)=A_{D}\left(z-D \gamma_{0} u\right)$

Theorem 5.1 For all $\rho \geq 0$, the PDE system (4.2) can be modeled abstractly as follows:

$$
y_{t}=\mathrm{A} y+\mathrm{B} g+\mathrm{G} w \in\left[\mathrm{D}\left(\mathrm{A}^{*}\right)\right]^{\prime}, y_{0} \in \mathrm{H}
$$

where A, B, G are defined as in (4.15) and (5.37). Moreover, $\mathrm{A}$ is the infinitesimal generators of s.c. semigroup $e^{\mathrm{A} t}$ on $\mathrm{H}$ with the domain $\mathrm{D}(\mathrm{A})$ defined in (4.16). When $\rho>0$ this semigroup is analytic.

This theorem follows from the results presented in the previous subsection. Regarding the analyticity of the semigroup generated by $\mathrm{A}$, when $\rho>0$, this follows from Theorem 5.2 on the strength of the fact that $\mathrm{A}$ in (5.38) is just a topologically equivalent representation of $\mathrm{A}$ in (4.15).

Our present goal is to verify that the control problem defined by $(A, B, G)$ satisfies the assumptions imposed by abstract theorem 3.1. We thus proceed next with verification of assumptions imposed on the control and observation operators.

\subsubsection{Verification of the Abstract Assumptions}

Control Operator B. The operator B defined in (5.40) is unbounded from $L_{2}\left(\Gamma_{s}\right)$ to $\mathcal{H}$ as the operator $A N$ is unbounded from $L_{2}\left(\Gamma_{s}\right)$ to $\mathrm{H}$. Likewise the operator $A_{D} D$ is unbounded. To apply Theorem 3.1 to (5.41), it is critical to verify the validity of Assumption (H.2) for the operator $B$.

Proposition 3.2 The resolvent $R(\lambda, \mathrm{A})$ satisfies $R(\lambda, \mathrm{A}) \mathrm{B} \in \mathrm{L}(\mathrm{U} \rightarrow \mathrm{H})$ for all $\lambda$ in the resolvent set of $\mathrm{A}$.

Proof. When $\rho=0$ it has been already shown in [36] that

$$
R(\lambda, \mathrm{A}) \mathrm{B} \in L(\mathrm{U} \rightarrow \mathrm{H}), \lambda>0
$$

We shall then consider now the case $\rho \neq 0$. Without loss of generality we may take $L_{f}=0$. Writing $R(\lambda, \mathrm{A}) \mathrm{B}\left(g_{0}, g_{1}\right)=(f, v, z)$ yields:

$$
\begin{aligned}
& A N_{1} g_{1}=A(f+N \sigma(v) v+\rho N \sigma(z) v)-\lambda f \\
& z=\lambda v \\
& \rho A_{D} D g_{0}=A_{D}\left(v+\rho z-D \gamma_{0} v-\rho D \gamma_{0} f\right)-\lambda z
\end{aligned}
$$

which is equivalent to

$$
\begin{aligned}
& A\left(f+N \sigma(v) v+\rho N \sigma(z)-N_{1} g_{1}\right)=\lambda f \\
& A_{D}\left(v+\rho z-D \gamma_{0} v-\rho D g_{0}-\rho D \gamma_{0} f\right)=\lambda z=\lambda^{2} v
\end{aligned}
$$

This amounts to solving the following system

$$
\begin{aligned}
& f-\lambda A^{-1} f+N \sigma(v+\rho \lambda v) v=N_{1} g_{1} \\
& v+\rho \lambda v-\lambda^{2} A_{D}^{-1} v-D \gamma_{0}(v+\rho f)=\rho D g_{0}
\end{aligned}
$$

Denoting $\zeta \equiv(1+\rho \lambda) v$ and applying the variational definition of the operators introduced yields

$$
\begin{aligned}
& (\sigma(f), \varepsilon(f))_{f}+\lambda|f|_{f}^{2}+\langle\sigma(\zeta) \cdot v, f\rangle=\left\langle g_{1}, f\right\rangle \\
& (\sigma(\zeta), \varepsilon(\zeta))_{s}+\lambda^{2}(v, \zeta)_{s}-\langle v+\rho f, \\
& \sigma(\zeta) \cdot v>=\left\langle g_{0}, \sigma(\zeta) \cdot v\right\rangle
\end{aligned}
$$

We also note that the second equation in (5.44) can be written as

$$
\operatorname{div} \sigma(\zeta)-\lambda^{2} v=0
$$

Taking inner product with $v$ gives

$$
\langle\sigma(\zeta) \cdot v, v\rangle=\lambda^{2}|v|_{s}^{2}+(\sigma(\zeta), \varepsilon(v))_{s}
$$

Now we are ready to decouple.

$$
\begin{aligned}
& \rho(\sigma(f), \varepsilon(f))_{f}+\rho \lambda|f|_{f}^{2}-\langle\sigma(\zeta) \cdot v, v> \\
& +(\sigma(\zeta), \varepsilon(\zeta))_{s}+\lambda^{2}(v, \zeta)_{s}=\left\langle g_{0}, \sigma(\zeta) \cdot v>+\rho\left\langle g_{1}, f>\right.\right.
\end{aligned}
$$

and using (5.47)

$$
\rho(\sigma(f), \varepsilon(f))_{f}+\lambda|f|_{f}^{2}-\lambda^{2}|v|_{s}^{2}-(\sigma(\zeta), \varepsilon(v))_{s}
$$




$$
+(\sigma(\zeta), \varepsilon(\zeta))_{s}+\lambda^{2}(v, \zeta)_{s}=\left\langle g_{0}, \sigma(\zeta) \cdot v\right\rangle+\rho\left\langle g_{1}, f\right\rangle
$$

In order to treat the term with $g_{0}$ we use once more (5.47)with $v$ replaced by $D g_{0}$

$\left.<\sigma(\zeta) \cdot v, g_{0}\right\rangle=\lambda^{2}\left(v, D g_{0}\right)_{s}+\left(\sigma(\zeta), \varepsilon\left(D g_{0}\right)\right)_{s}$

Combining (5.49) and (5.50) gives

$\rho(\sigma(f), \varepsilon(f))_{f}+\rho \lambda|f|_{f}^{2}+(\sigma(\zeta), \varepsilon(\zeta-v))_{s}+\lambda^{2}(v, \zeta-v)_{s}$

$=\rho<g_{1}, f>+\lambda^{2}\left(v, D g_{0}\right)_{s}+\left(\sigma(\zeta), \varepsilon\left(D g_{0}\right)\right)_{s}$

and going back to the definition of $\zeta$, hence $\zeta-v=\rho \lambda v$

$\rho(\sigma(f), \varepsilon(f))_{f}+\rho \lambda|f|_{f}^{2}+\rho \lambda(1+\rho \lambda)(\sigma(v), \varepsilon(v))_{s}+\rho \lambda^{3}(v, v)_{s}$

$=\rho<g_{1}, f>+\lambda^{2}\left(v, D g_{0}\right)_{s}+\left(\sigma(\zeta), \varepsilon\left(D g_{0}\right)\right)_{s}$

Korn's inequality and elliptic regularity $D \in L\left(H^{1 / 2}(\Gamma) \rightarrow H^{1}(\Omega)\right)$ then gives

$\rho|f|_{1, \Omega_{f}}^{2}+\rho \lambda|f|_{f}^{2}+\lambda(1+\rho \lambda)|v|_{1, \Omega_{s}}^{2}+\rho \lambda^{3}|v|_{s}^{2}$

$\leq \varepsilon|f|_{1, \Omega_{f}}^{2}+C_{\varepsilon}\left|g_{1}\right|_{-1 / 2, \Gamma_{s}}^{2}+\varepsilon(1+\rho \lambda)^{2}|v|_{1, \Omega_{s}}^{2}$

$+C_{\varepsilon}\left|g_{0}\right|_{1 / 2, \Gamma_{s}}^{2}+\varepsilon \lambda^{4}|v|_{s}^{2}+C_{\varepsilon}\left|g_{0}\right|_{-1 / 2, \Gamma_{s}}^{2}$

Selecting small $\varepsilon$ we obtain that for $g=\left(g_{0}, g_{1}\right) \in H^{1 / 2}\left(\Gamma_{s}\right) \times H^{-1 / 2}\left(\Gamma_{s}\right)$ one has

$(f, v, z) \in\left(H^{1}\left(\Omega_{f}\right) \cap H\right) \times H^{1}\left(\Omega_{s}\right) \times H^{1}\left(\Omega_{s}\right)$

Disturbance Operator $\mathbf{G}$ The operator $G$ defined in (5.37) involving localized function $\phi$ is bounded from $L_{2}\left(\Omega_{f}\right) \times L_{2}\left(\Omega_{s}\right)$ to $\mathrm{H}$, thus it automatically satisfies Assumption (H.3) and (H.6) for any bounded operator $R$ with $\mathrm{D}(R) \supset \mathrm{H}$. As for the component $A N$ of disturbance operator, we shall have the same singular estimate that is discussed below. The explicit form of the adjoint to the disturbance operator $\mathrm{G}$ is given by

$\mathrm{G}^{*}(u, v, z)=\left[\varphi_{\omega_{f}} u, \varphi_{\omega_{s}} z,\left.u\right|_{\Gamma_{s_{2}}}\right]$

\subsubsection{Singular Estimate Property}

We now investigate the dynamical property of the pair $\{A, B\}$ which is critical for applying Theorem 3.1 to the fluid-structure interaction model. Since the control operator $\mathrm{B}$ is not bounded, the task is not trivial. The main property to be verified is Output Singular Estimate Property Hypotheses (H.5) and (H.6) in Theorem 3.1. The Singular Estimate provides for quantitative measure of the unboundedness of control operator. To this end, we define the following scale of Hilbert spaces parameterized by the parameter $\delta \geq 0$ :
$\mathrm{H}_{-\delta} \equiv \mathrm{H} \times H^{1-\delta}\left(\Omega_{s}\right) \times H^{-\delta}\left(\Omega_{s}\right), \quad \delta \geq 0$

We then remark that with the above notation we have: $\mathrm{H}=\mathrm{H}_{0}$, where $\mathcal{H}$ is the energy space defined in (??). The following result is critical.

Theorem 5.3. 1 . If $g_{0}=0$ and $\rho \geq 0$, the semigroup $e^{\mathrm{A} t}$ generated in $\mathrm{H}$ by the operator $\mathrm{A}$ in (4.15) and the control operator B in (5.37) satisfy the the following Singular Estimate (SE)

$\left|e^{\mathrm{A} t} \mathrm{~B}\left(0, g_{1}\right)\right|_{\mathrm{H}_{-\delta}} \leq \frac{C_{T}}{t^{1 / 4+\varepsilon}}\left|g_{1}\right|_{L_{2}\left(\Gamma_{s 1}\right)}, \quad 0<t \leq T$

for every $g_{1} \in L_{2}\left(\Gamma_{s 1}\right)$, any $\delta>0$, any $\varepsilon>0$ and any $\rho \geq 0$.

2. If $g_{0} \neq 0$, then with $\rho>0$ the semigroup $e^{\mathrm{A} t}$ generated in $\mathrm{H}$ by the operator $\mathrm{A}$ in (5.38) and the control operator $B$ in (5.40) satisfy the following Singular Estimate (SE)

$\left|e^{\mathrm{A} t} \mathrm{~B}\left(g_{0}, g_{1}\right)\right|_{H} \leq \frac{C_{T}}{t^{3 / 4+\varepsilon}}\left(\left|g_{1}\right|_{H^{-1 / 2}\left(\Gamma_{s 1}\right)}+\left|g_{0}\right|_{H^{1 / 2}\left(\Gamma_{s}\right)}\right), \quad 0<t \leq T(5.57)$

for any $\varepsilon>0$

Remark 5.1 We note that the singularity of the control operator B in both Neumann and Dirichlet case is consistent with singularity established for parabolic dynamics subject to Neumann (resp. Dirichlet) boundary controls [21-23, 26, $37,38]$. The only difference is that in the Dirichlet case the present coupled model requires more regular control operators $g_{0} \in H^{1 / 2}(\Gamma)$ rather than $L_{2}(\Gamma)$. This is due to unbounded coupling.

Proof. Step 1: Structure of the adjoint $B^{*}$. We consider operator $\mathrm{B}: \mathrm{U} \rightarrow \mathrm{H}$ given by (5.40). For any $y=(u, v, z) \in D(\mathrm{~A})$ we have

$\left(\mathrm{B}\left(g_{0}, g_{1}\right), y\right)_{\mathrm{H}}=\left(A N_{1} g_{1}, u\right)_{\Omega_{f}}$

$+\rho\left(A_{D} D g_{0}, z\right)_{\Omega_{s}}=<g_{1}, N_{1}^{*} A u>+\rho<g_{0}, D^{*} A_{D} z>$

$=\left\langle g_{1},\left.u\right|_{\Gamma_{s}}>+\rho\left\langle\Lambda_{\tau}^{1 / 2} g_{0}, \Lambda_{\tau}^{-1 / 2} \sigma(z) \cdot v>\right.\right.$

$=<<\left(g_{1}, g_{0}\right),\left(\left.u\right|_{\Gamma_{s}}, \rho \Lambda_{\tau}^{-1} \sigma(z) \cdot v>>_{\mathrm{U}}\right.$

where $\Lambda_{\tau}$ is first order tangential operator and we have used the identifications

$N_{1}^{*} A u=\left.u\right|_{\Gamma_{1 s}}, D^{*} A_{D} z=\sigma(z) \cdot v$

The above implies:

$\mathrm{B}^{*} y=\left[\mathrm{B}_{1}^{*} y, \mathrm{~B}_{2}^{*} y\right]=\left[\left.u\right|_{\Gamma_{1 s}}, \rho \Lambda_{\tau}^{-1} \sigma(z) \cdot v\right]$

By appealing to (5.58) inequality in (5.56) in equivalent to show

$\left|\mathrm{B}_{1}^{*} e^{\mathrm{A}^{*} t} Y\right|_{L_{2}\left(\Gamma_{s}\right)} \leq C|Y|_{\mathrm{H}}$

Where we denote $\mathrm{B}_{1}(g) \equiv \mathrm{B}(0, g)$. We provide PDE representation of this statement. 
Consider the system

$\left\{\begin{array}{l}u_{t}=A u-L_{f} u-A N\left(\sigma\left(v+\rho v_{t}\right) \cdot v\right) \\ v_{t t}=\operatorname{div} \sigma\left(v+\rho v_{t}\right) \\ v_{t}=u \text { on } \Gamma_{s} \\ u(0)=u_{0}, v(0)=v_{0}, v_{t}(0)=v_{1}\end{array}\right.$ (5.58)

With initial condition $Y=\left(u_{0}, v_{0}, v_{1}\right) \in \mathrm{H}$. Recalling $\mathrm{B}_{1}^{*} e^{\mathrm{A}^{*} t} Y=-\gamma_{0}(u(t))$

where $\left(u, v, v_{t}\right)$ satisfies (5.60). Thus, the adjoint operator to $B_{1}$ is identified with the trace of solution. The first part of Theorem 5.3 (When $\rho=0$ ) follows from regularity decomposition results in [36], Theorem 5.1. The second part of this theorem $(\rho>0)$ uses the analyticity of the semigroup along with a characterization of the fractional powers. The details are given below. The trace function corresponding to (5.60) can be written as

$\gamma_{0}(u(t))=N_{1}^{*} A e^{\left(A-L_{f}\right) t} Y+N_{1}^{*} A \int_{0}^{t} e^{\left(A-L_{f}\right)(t-s)}$

$A N\left(\left[\sigma\left(v(s)+\rho v_{t}(s)\right] \cdot v\right) d s\right.$

\section{Step 2: Proof of Theorem 5.3 Part (i):}

Case $1 \rho=0$. In this case we need only to estimate $B_{1}^{*}$ associated with $\gamma_{0}(u(t))$ (Since $g_{0}=0$ ). The following regularity result has been proved in [36].

Lemma 5.4 Let $\rho=0$. Then for every $\delta>0$ the following decomposition holds $v=v_{1}+v_{2}$ where

$\left|\sigma\left(v_{1}\right)\right|_{H^{\delta}\left((0, T), L_{2}\left(\Gamma_{s}\right)\right)} \leq C|Y|_{H_{\delta}},\left|\sigma\left(v_{2}\right)\right|_{C\left([0, T], H^{-1 / 2}\left(\Gamma_{s}\right)\right)} \leq C|Y|_{H}$

Taking $\rho=0$ in (5.62) and using

$A^{1 / 2} N \subset \mathrm{L}\left(H^{-1 / 2}\left(\Gamma_{s}\right) \rightarrow L_{2}\left(\Omega_{f}\right)\right)$

along with the analyticity of $A$

$\left|N_{1}^{*} A e^{A t} A^{\alpha} u\right|_{L_{2}\left(\Gamma_{s}\right)} \leq \frac{C|u|_{L_{2}\left(\Omega_{f}\right)}}{t^{\alpha+1 / 4+\varepsilon}}$

one obtains

$$
\begin{aligned}
& \left|N_{1}^{*} A \int_{0}^{t} e^{\left(A-L_{f}\right)(t-s)} A^{1 / 2}\left(A^{1 / 2} N\left[\sigma\left(v_{1}(s)\right) \cdot v\right]\right) d s\right| \\
& \leq C \int_{0}^{t}(t-s)^{-1 / 2-1 / 4-\varepsilon}\left|A^{1 / 2} N\left[\sigma\left(v_{1}(s)\right) \cdot v\right]\right|_{L_{2}\left(\Gamma_{s}\right)} d s \\
& \leq C\left|\sigma\left(v_{1}(\cdot)\right)\right|_{C\left([0, T], H^{-1 / 2}\left(\Gamma_{s}\right)\right)} \leq C|Y|_{H}
\end{aligned}
$$

The contribution of $v_{2}$ is evaluated in a similar manner by using the fact that for all $\delta>0$ there exists $p>2$

$H^{\delta}(0, T) \subset L_{p}(0, T)$

and for $p>2$ the convolution

$t^{1 / 2+\varepsilon} \circ L_{p} \subset L_{\infty}(0, T)$
This completes the proof when $\rho=0$.

Case $2 \rho>0$. In this case we have analyticity of the generator corresponding to the operator A. This means, in particular,

$$
\left|\mathrm{A}^{\alpha} e^{\mathrm{A} t}\right|_{\mathrm{L}(\mathrm{H})} \leq \frac{C}{t^{\alpha}}, \quad \alpha \in[0,1]
$$

The next step is to use the characterization of the domain of fractional powers of $\mathrm{A}$.

$$
\begin{aligned}
& \mathrm{D}\left(\mathrm{A}^{1 / 2}\right)=\left\{(u, v, z) \in \mathrm{H}, u \in H^{1}\left(\Omega_{f}\right),\right. \\
& \left.v \in H^{1}\left(\Omega_{s}\right), z \in H^{1}\left(\Omega_{s}\right), z=u \text { on } \Gamma_{s}\right\}
\end{aligned}
$$

Since

$\mathrm{B}_{1}^{*} e^{\mathrm{A}^{*} t} Y=\left.\gamma(u(t)) \equiv u(t)\right|_{\Gamma_{s}}$

We can write on the dynamics

$\mathrm{B}_{1}^{*} e^{\mathrm{A}^{*} t} Y=\left.\gamma\left(v_{t}(t)\right) \equiv v_{t}(t)\right|_{\Gamma_{s}}$

Using trace theorem

$\left|\mathrm{B}_{1}^{*} e^{\mathrm{A}^{*} t} Y\right|_{L_{2}\left(\Gamma_{s}\right)}=\left|\gamma\left(v_{t}(t)\right)\right|_{L_{2}\left(\Gamma_{s}\right)} \leq\left|v_{t}(t)\right|_{H^{1 / 2+\varepsilon}\left(\Omega_{s}\right)}$

$\leq\left|\mathrm{A}^{1 / 4+\varepsilon} Y(t)\right|_{\mathrm{H}} \leq \frac{C}{t^{1 / 4+\varepsilon}}|Y(t)|_{\mathrm{H}}$

Here $Y(t)=e^{\mathrm{A} t} Y$, the solution to the equation system (5.1) with $g_{0}=0$.

Step 3: Proof of Theorem 5.3. Part (ii) Case $\rho>0, g_{0} \neq 0$.

Here we shall use the calculations from given for the proof of the resolvent estimate (5.42). Indeed, we already know that

$$
R(\lambda, \mathrm{A}) \mathrm{B} \in \mathrm{L}\left(\mathrm{U} \rightarrow H^{1}\left(\Omega_{f}\right) \cap \mathbf{H} \times H^{1}\left(\Omega_{s}\right) \times H^{1}\left(\Omega_{s}\right)\right.
$$

The latter space is compatible topologically with $D\left(\mathrm{~A}^{1 / 2}\right)$ modulo Dirichlet boundary conditions which are embedded in the definition of. On the other hand, boundary conditions are not recognized below $H^{1 / 2}(\Omega)$ scale. We also have (due to the analyticity of the semigroup, $[21,39,40]$.

$$
D\left(\mathrm{~A}^{\theta}\right)=\left[D\left(\mathrm{~A}^{1 / 2}\right), \mathrm{H}\right]_{2 \theta}, \theta \in[0,1 / 2]
$$

For $\theta<1 / 2$ For $\theta<1 / 4$ Dirichlet boundary conditions $z=u$, on $\Gamma_{s}$ are no longer recognized (due to density) of $H_{0}(\Omega)$ in $H^{1 / 2}(\Omega)$ we then have

$$
D\left(\mathrm{~A}^{\theta}\right)=\left[D\left(\mathrm{~A}^{1 / 2}, \mathrm{H}\right]_{2 \theta}=H^{\theta}\left(\Omega_{f}\right) \cap \mathbf{H} \times H^{1}\left(\Omega_{s}\right) \times H^{\theta}\left(\Omega_{s}\right)\right.
$$

Thus,

$$
R(\lambda, \mathrm{A}) \mathrm{B} \in \mathrm{L}\left(\mathrm{U} \rightarrow D\left(\mathrm{~A}^{\theta}\right)\right), \text { for } \theta<1 / 4
$$

Analyticity of the semigroup then implies 


$$
\begin{aligned}
& \left|e^{\mathrm{A} t} \mathrm{~B}\right|_{L\left(H^{1 / 2}(\Gamma) \rightarrow H\right.} \leq\left|\mathrm{A} R(\lambda, \mathrm{A}) e^{\mathrm{A} t} \mathrm{~B}\right|_{L\left(H^{1 / 2}(\Gamma) \rightarrow H\right.} \\
& =\left|\mathrm{A}^{1-\theta} \mathrm{A}^{\theta} R(\lambda, \mathrm{A}) e^{\mathrm{A} t} \mathrm{~B}\right|_{L\left(H^{1 / 2}(\Gamma) \rightarrow H\right.} \leq\left|\mathrm{A}^{1-\theta} e^{\mathrm{At}} \mathrm{A}^{\theta} R(\lambda, \mathrm{A}) \mathrm{B}\right|_{L\left(H^{1 / 2}(\Gamma) \rightarrow H\right.} \\
& \leq\left|\mathrm{A}^{1-\theta} e^{\mathrm{A} t}\right|_{L(\mathrm{H})} \leq C t^{-1+\theta}, \theta<1 / 4
\end{aligned}
$$

Which is a desired singular estimate estimate.

Remark 5.2 We note a potential loss in the singular estimate $(\theta<1 / 4$, rather than $\theta \leq 1 / 2)$ which results from accounting on compatibility conditions characterizing the domain of the generator. This prevents us full exploitation of $H^{1}$ regularity for the domain of $\mathrm{A}^{1 / 2}$.

From Theorem 5.3, we are ready to obtain the soughtafter Output Singular Estimate property for the pair $\{A, B\}$.

Corollary 5.5 Consider the PDE system (4.2) and corresponding abstract model (5.41) satisfying, in particular, Theorem 5.3, with $\delta>0$ abitrarily and henceforth fixed. Let $\rho \geq 0$.

[Part I] Let $g_{0}=0$ (Neumann control only). Assume that the observation operator $\mathrm{R}$ satisfies

$\mathrm{R} \in \mathrm{L}\left(\mathrm{H}_{-\delta} ; \mathrm{Z}\right)$

where $\mathcal{Z}$ is the observation or output space (possibly $\mathrm{H}=\mathrm{Z}$ ). Then (5.56) yields the desired Output Singular Estimate

$$
\left|\mathrm{R} e^{\mathrm{At}} \mathrm{B} g\right|_{\mathrm{Z}} \leq \frac{C_{T}}{t^{1 / 4+\varepsilon}}|g|_{L_{2}\left(\Gamma_{s}\right)}, \quad 0<t \leq T .
$$

[Part II] In the viscoelastic case, (still with $g_{0}=0$ ) when $\rho>0$ one can take $\delta=0$. In addition, $\mathrm{R}$ can be unbounded $\mathrm{A}^{-\theta} \mathrm{R} \in \mathrm{L}(\mathrm{H}), \theta<\frac{3}{4}$, in which case one has

$\left|\mathrm{R} e^{\mathrm{A} t} \mathrm{~B} g\right|_{\mathrm{Z}} \leq \frac{C_{T}}{t^{1 / 4+\theta+\varepsilon}}|g|_{L_{2}\left(\Gamma_{s}\right)}, \quad 0<t \leq T$.

[Part III] Let $\rho>0$ and $g_{0} \neq 0$ (Dirichlet control). Then

$\left|\mathrm{R} e^{\mathrm{At}} \mathrm{B} g\right|_{Z} \leq \frac{C_{T}}{t^{3 / 4+\varepsilon}}|g|_{H^{1 / 2}\left(\Gamma_{s}\right)}, \quad 0<t \leq T$.

for any R satisfying (5.75) with $\delta=0$.

Next, we illustrate examples of the observation operator $\mathrm{R}$ satisfying the smoothing property (5.75), (5.76) in Corollary 4.5.

Example 5.1: Let $v_{1} \in H^{1-\delta}\left(\Omega_{s}\right), \quad v_{2} \in H^{-\delta}\left(\Omega_{s}\right), \quad \delta>0$ small. Define the operators $\mathrm{R}_{1}$ and $\mathrm{R}_{2}$ on $v_{1}$ and $v_{2}$ respectively by

$\mathrm{R}_{1} v_{1}=\left(v_{1}, \varphi_{1}\right) \psi_{1} ; \mathrm{R}_{2} v_{2}=\left(v_{2}, \varphi_{2}\right) \psi_{2}$

where $\phi_{i}, \psi_{i}$ are fixed vectors:

$\varphi_{1} \in\left[H^{1-\delta}\left(\Omega_{s}\right)\right]^{\prime} ; \psi_{1} \in H^{1}\left(\Omega_{s}\right) ; \varphi_{2} \in H^{\delta}\left(\Omega_{s}\right) ; \psi_{2} \in L_{2}\left(\Omega_{s}\right)$

Thus, $\mathrm{R}_{1}$ is bounded (in fact smoothing), $\mathrm{R}_{1} \in \mathrm{L}\left(H^{1-\delta}\left(\Omega_{s}\right) ; H^{1}\left(\Omega_{s}\right)\right)$, but not smoothing above $H^{1}\left(\Omega_{s}\right)$.
Similarly, $\quad \mathrm{R}_{2}$ is bounded (thus smoothing): $H^{-\delta}\left(\Omega_{s}\right) \rightarrow L_{2}\left(\Omega_{s}\right), \quad \mathrm{R}_{2} \in \mathrm{L}\left(H^{-\delta}\left(\Omega_{s}\right) ; L_{2}\left(\Omega_{s}\right)\right), \quad$ but not smoothing above $L_{2}\left(\Omega_{s}\right)$. Finally, for $\left\{u_{1}, v_{1}, v_{2}\right\} \in \mathrm{H} \times H^{1-\delta}$ $\left(\Omega_{s}\right) \times H^{-\delta}\left(\Omega_{s}\right)$, define $\mathrm{R} \in \mathrm{L}\left(\mathrm{H}_{-\delta} ; \mathrm{H}\right) \quad$ (but not smoothing above $\mathcal{H}$ ) by:

$\mathrm{R}\left[\begin{array}{c}u_{1} \\ v_{1} \\ v_{2}\end{array}\right]=\left[\begin{array}{ccc}I_{f} & 0 & 0 \\ 0 & \mathrm{R}_{1} & 0 \\ 0 & 0 & \mathrm{R}_{2}\end{array}\right]=\left[\begin{array}{c}u_{1} \\ \mathrm{R}_{1} v_{1} \\ \mathrm{R}_{2} v_{2}\end{array}\right] \in \mathrm{H} \equiv \mathrm{H} \times H^{1}\left(\Omega_{s}\right) \times L_{2}\left(\Omega_{s}\right)$

Example 5.2: On $\Omega_{s}$, let

$\bar{\Delta}_{N} f=(-\Delta) f, \quad \mathrm{D}\left(\bar{\Delta}_{N}\right)=\left\{f \in H^{2}\left(\Omega_{s}\right):\left.\frac{\partial f}{\partial v}\right|_{\partial \Gamma_{s}}=0\right\}$

So $\quad$ that $\mathrm{D}\left(\bar{\Delta}_{N}^{1 / 2}\right)=H^{1}\left(\Omega_{s}\right), \quad \mathrm{D}\left(\bar{\Delta}_{N}^{\delta / 2}\right)=H^{\delta}\left(\Omega_{s}\right), \delta \geq 0$. Let $\left\{u_{1}, v_{1}, v_{2}\right\} \in \mathrm{H}_{-\delta}$, so that, by $(1.3 \mathrm{~b}), \quad v_{1} \in H^{1-\delta}\left(\Omega_{s}\right)$ and $v_{2} \in H^{-\delta}\left(\Omega_{s}\right)$. Thus,

$\bar{\Delta}_{N}^{-\delta / 2} v_{1} \in H^{1}\left(\Omega_{s}\right), \quad \bar{\Delta}_{N}^{-\delta / 2} v_{2} \in L_{2}\left(\Omega_{s}\right)$

Thus, the operator $\mathrm{R} \in \mathrm{L}\left(\mathrm{H}_{-\delta} ; \mathrm{H}\right)$ defined by

$$
\mathrm{R}\left[\begin{array}{c}
u_{1} \\
v_{1} \\
v_{2}
\end{array}\right]=\left[\begin{array}{ccc}
I_{f} & 0 & 0 \\
0 & \bar{\Delta}_{N}^{-\delta / 2} & 0 \\
0 & 0 & \bar{\Delta}_{N}^{-\delta / 2}
\end{array}\right]\left[\begin{array}{c}
u_{1} \\
v_{1} \\
v_{2}
\end{array}\right]=\left[\begin{array}{c}
u_{1} \\
\bar{\Delta}_{N}^{-\delta / 2} v_{1} \\
\bar{\Delta}_{N}^{-\delta / 2} v_{2}
\end{array}\right] \in \mathrm{H}
$$

where $I_{f}$ is the identity on $\mathbb{H}$ and satisfies property (5.75) with respect to the output space $\mathrm{Z}=\mathrm{H}$. In this example, $\mathrm{R}$ is also smoothing above $\mathcal{H}$, unlike the case of Example 5.1.

Remark 5.3 In the viscoelastic case, $\rho>0$, one can take $\delta=0$. In fact, not only every bounded observation $\mathrm{R}$ satisfies the hypotheses. but $\mathrm{R}$ can also be unbounded up up to the order of $3 / 4$. In particular $R Y=\left(\nabla u, \nabla v, \nabla v_{t}\right)$.

$\vec{F}, \vec{F} \in \mathrm{H}^{3}$ is an example of the observation complying with the hypotheses. This observation allows to measure the stresses in addition to displacements.

From the above analysis, we have verified that the operator A, B and G defined in (4.15) satisfy the Assumptions (H.1)-(H.3). Thus we conclude that the fluidstructure interaction model (4.2) satisfies the Assumptions of Theorem 3.1 with $\alpha=1 / 4+\varepsilon$ for the Neumann case and with $\alpha=3 / 4+\varepsilon$ for the Dirichlet case. Applying Theorem 3.1 leads to all the statements in the final result Theorem 4.1.

Remark 5.4 The theory presented above, with $\rho>0$, does not require any smoothing property imposed on the observation operator R. In fact, one can also consider unbounded observation (eg first order differential operator in the Neumann case). This is in contrast with other treatments where such smoothing was required $[25,35,36]$.

\section{CONCLUSIONS AND SOME OPEN PROBLEMS}

We have studied the min-max game theory problem of a linear fluid-structure interaction model, with control and disturbance acting on the interface between the two media. 
(disturbance may also be scattered in two media, which is a mathematically easier problem). Our analysis was a specialization, to the present model, of an abstract theory where the essential features of the model are singled out. The model is characterized by an Output Singular Estimate involving both control and disturbance, as well as a "minimally smoothing" observation operator. These singular estimates (which are automatically satisfied for the system consisting of just one parabolic PDE, which generates an analytic semigroup) reflects the influence of the parabolic component (the fluid) onto the overall coupled system. Both elastic and viscoelastic responses of the structure are considered. It is shown that viscoelastic model is associated with an analytic semigroups. As a consequence, a larger range of disturbances and observations can be treated. The theory presented is optimal and complete and yields the sought-after saddle-point control and disturbance solutions in a pointwise feedback form, through the critical role of a gain operator defined in terms of a Riccati operator, which is the solution of a non-standard Riccati differential equation.

Open Problems. Regarding the present min-max game theory for fluid-structure interaction model, we may list the following open problems:

1. So far we have established the wellposedness of the associated non-standard Riccati differential equation, whose positive self-adjoint solution provides the solution of the min-max problem in pointwise feedback form. A natural next step is to construct numerical methods for solving this Riccati differential equation. This is a very important aspect of the present research program with stimulus coming from applications to actual design [41, 42]. Computational methods for algebraic Riccati equations, with due convergence theory, have a long history in the finite dimensional setting [43]. They arise as culmination in the study of optimal control problems over an infinite time horizon; thus with only one player, the control. The numerical theory for infinite dimensional standard algebraic Riccati equations (as they arise from PDE) has also been available since 80 's: [26, 22] in the parabolic case and [44] in the hyperbolic case. However, in the case of Riccati differential equations - let alone the non-standard Riccati differential equations arising in min-max game theory - the literature is very meager at best. It is a fertile ground for further research.

2. The model considered depends on a linear operator $L_{f}(u)$ which results from linearization of Navier Stokes equation around some static equilibrium. It will be interesting to consider the force $f$ as a control function (for instance radiation force in an ultrasound). This will lead to an interesting bilinear control problem. Indeed, the control operator $B_{0}(f, u)=\nabla u \cdot y_{e}+u \cdot \nabla y_{e}$ is bilinear. As shown in Lemma 5.1 the control operator $B_{0}(f, u): L_{2}\left(\Omega_{f}\right)$ $1 \times E \rightarrow L_{2}\left(\Omega_{f}\right)$. Thus, the control operator while is not bounded with respect to $u \in \mathrm{H}$, it is bounded with respect to $f \in L_{2}(\Omega)$. Exploiting the latter boundedness leads to an optimistic conclusions regarding the realizability of control as a feedback operator.
3. The result presented in Theorem 5.3 requires $\delta>0$ in the purely elastic case $\rho=0$. This is satisfied when the observation $R$ represents some incremental "smoothing effect". It will be interesting to obtain the results with $\delta=0$. The problem is related to the validity of "singular estimate". It turns out that such estimate is not valid with $\delta=0$. However, the "defect" of that estimate has some hyperbolic properties that are "controllable". Based on this, the theory of optimal control and associate Riccati equations has been constructed in [45]. It is reasonable to expect that such theory could also be developed for the mini-max game problem studied in this paper [46]. However, this theory is not expected to recover a full boundedness of the gain operator $B^{*} P(t)$. Due to hyperbolic propagation effects, this operator will retain some of the singularity of the dynamics. However, the Riccati equation will still be well defined on $D(A)$.

\section{CONFLICTS OF INTEREST}

The authors confirm that this article content has no conflicts of interest.

\section{ACKNOWLEDGEMENTS}

Declared none.

\section{REFERENCES}

[1] von Neumann J. Zur Theorie der Gesellschaftsspiele. Math Ann 1928; 100(1): 295-320.

[2] Isaacs R. Differential Games. New York: John Wiley and Sons 1965.

[3] Berkovitz L. Lectures on differential games. In: Kuhn HW, Szego GP, Eds. Differential Games and Related Topics. North Holland: North Holand Publishers 1971; pp. 3-45.

[4] Friedman A. Differential Games. New York: Wiley-Interscience 1971.

[5] Myerson R. Game Theory: Analysis of Conflict. USA: Harvard University Press 1991

[6] Delfour MC, Mitter SK. Reachability of perturbed systems and min sup problems. SIAM J. Control Optim 1969; 7: 51-69.

[7] Bernhard P. Linear-quadratic, two-person, zero-sum differential games: necessary and sufficient conditions. J Optim Theory Appl 1979; 27: 51-69.

[8] Basar T, Bernhard P. $H^{\infty}$-optimal control and related minimax design problems: a dynamic game approach. Basel: Birkhäuser 1991.

[9] McMillan C, Triggiani R. Min-Max game theory and algebraic Riccati equations for boundary control problems with analytic semigroups, Part I: The stable case. Marcel Dekker Lecture Notes Pure Appl Math 1994; 152: 757-80.

[10] McMillan C, Triggiani R. Min-Max game theory and algebraic Riccati equations for boundary control problems with analytic semigroup, Part II: The general case. J Nonlinear Anal 1994; 22: 431-65.

[11] Lions JL. Quelques methods de resolution des problemes aux limits nonlinearies. Dunod. Paris 1969.

[12] Du Q, Gunzburger MD, Hou LS, Lee J. Analysis of a linear fluidstructure interaction problem. Discrete Contin Dyn Syst 2003; 9(3): 633-50.

[13] Canic S, Mikelic A, Tambaca J. A two-dimensional effective model describing fluid-structure interaction in blood flow: analysis, simulation and experimental validation. Compte Rendus Mechanique Acad Sci Paris 2005; 333(12): 867-83.

[14] Canic S, Lamponi D, Mikelic A, Tambaca J. Self-consistent effective equations modeling blood flow in medium-t-large compliant arteries. Multiscale Model Simul 2005; 3(3): 559-96.

[15] Vito RP, Dixon S. Blood vessel constitutive models. Ann Rev Biomed Eng 2003; 5: 413-39. 
[16] Humphrey JD. Mechanics of the arterial wall: review and new directions. Crit Rev Biomed Eng 1995; 23: 1-62.

[17] Fung YC. Biomechanics: Mechanical Properties of Living Tissues. $2^{\text {nd }}$ ed. New York: Springer 1993.

[18] Constantin P, Foias C. Navier Stokes Equations. Chicago Lectures in Mathematics. Chicago University Chicago Press 1988.

[19] Moubachir M, Zolesio J-P. Moving shape analysis and control: Applications to Fluid Structure Interactions. London: Chapman and Hall/CRC 2006.

[20] Bociu L, Zolesio J-P. Linearization of a coupled system of nonlinear elasticity and viscous fluid. Modern Aspects of the Theory of Partial Differential Equations. Operator Theory. Adv Appl 2011; 216: 93-120.

[21] Bensoussan A, DaPrato G, Delfour M, Mitter S. Representation and Control of Infinite Dimensional Control Systems. Basel: Birkhauser, 1993.

[22] Lasiecka I, Triggiani R. Differential and Algebraic Riccati Equations with Applications to Point-Boundary Control Problems. In: Lecture Notes in Mathematics. vol. 164. Berlin: Springer Verlag 1991.

[23] Lasiecka I, Triggiani R. Control theory for partial differential equations: continuous and approximation theories. vol II: Abstract Hyperbolic systems over a finite time horizon, Encyclopedia of Mathematics and its Appications 75. Cambridge: Cambridge University Press 2000.

[24] Triggiani R, Zhang J. Min-max game theory and non-standard differential Riccati equations under singular estimates for $e^{A t} G$ and $e^{A t} G$ in the absence of analyticity. Set-Valued and Variational Analysis. Berlin: Springer-Verlag 2009; 17: 245-83.

[25] Lasiecka I, Triggiani R, Zhang J. The Fluid-Structure Interaction Model with Both Control and Disturbance at the Interface: A Game Theory Problem. Applicable Anal 2011; 90(6): 971-1009.

[26] Lasiecka I, Triggiani R. Control Theory for Partial Differential Equations: Continuous and Approximations Theories, vol I: Abstract Parabolic systems, Encyclopedia of Mathematics and its Appications 74. Cambridge: Cambridge University Press 2000.

[27] Triggiani R. Min-Max game theory and optimal control with indefinite cost under a singular estimate for $e^{A t} B$ in the absence of analyticity. In: Lorenzi A, Ruf B, Eds. Evolution Equations, Semigroups and Functional Analysis. Basel: Birkhäuser 2002; pp. 353-80.

[28] Triggiani R. Min-max game theory and non-standard differential Riccati equations for abstract hyperbolic-like equations. J Nonlinear Anal A Spec Issue 2012; 75: 1572-91.

[29] Kesavan S. Topics in Functional Analysis and Applications. New York: John Wiley and Sons 1989.

[30] Avalos G, Triggiani R. The coupled PDE-system arising in fluidstructure interaction. Part I: Explicit semigroup generator and its spectral properties. AMS Contemp Math Fluids Waves 2007; 440: $15-55$.
[31] Avalos G, Triggiani R. Semigroup well-posedness in the energy space of a parabolic-hyperbolic coupled Stokes-Lamé PDE system. Discrete Contin Dyn Syst 2009; 2(3): 417-48.

[32] Avalos G, Triggiani R. Boundary feedback stabilization of a coupled parabolic-hyperbolic Stokes-Lamé PDE system, J Evol Equ 2009; 9: 341-70.

[33] Barbu V, Grujic Z, Lasiecka I, Tuffaha A. Weak solutions for nonlinear fluid-structure interaction. AMS Contemp Math: Recent Trends in App Anal 2007; 440: 55-81.

[34] Barbu V, Grujic Z, Lasiecka I, Tuffaha A. Smoothness of Weak solutions to a nonlinear fluid-structure interaction model. Indiana $J$ Math 2008; 57(3): 1173-207.

[35] Lasiecka I, Tuffaha A. Riccati Equations for the Bolza Problem arising in boundary/point control problems governed by $C_{0}$ semigroups satisfying a singular estimate. J Optim Theory Appl 2008; 136: 229-46.

[36] Lasiecka I, Tuffaha A. Riccati theory and singular estimates for a Bolza control problem arising in linearized fluid-structure interaction. Syst Control Lett 2009; 58: 499-509.

[37] Lasiecka I. Optimal control problem and Riccati equation for system with unbounded controls and partially analytic generatorsApplications to boundary and point control problems. In: Functional Analytic Methods for Evolution Equations, Lecture Notes in Mathematics. Berlin: Springer 2004; vol. 1855: pp. 31369.

[38] Bucci F, Lasiecka I, Triggiani R. Singular estimate and uniform stability of coupled systems of hyperbolic/parabolic PDEs. Abstract Appl Anal 2002; 7: 169-237.

[39] Pazy A. Semigroup of Linear Operators Applications to Partial Differential Equations. New York: Springer 1979.

[40] Lunardi A. Abstract quasilinear parabolic equations. Math Ann 1984; 267: 395-415.

[41] Glowinski R, Pan TW, Periaux J. A fictious domain method for external incompressible viscous flow modeled by Navier-Stokes equation. Comput Methods Appl Mech Eng 1994; 112: 133-48.

[42] Politis AK, Stavvropoulus GP, Christolis C. Numerical modelling of simulated blood flow in idealized composite arterial coronary grafts: Transient flow. J Biomech 2008; 41: 25-39.

[43] Bini D, Iannazzo B, Meini B. Numerical solution of algebraic Riccati equations. In: SIAM Fundamentals of Algorithm 2011; 9.

[44] Hendrickson E, Lasiecka I. Numerical approximations and regularizations of Riccati equations arising in hyperbolic dynamics with unbounded control operators. Comput Optim Appl 1993; 2: 343-90.

[45] Acquistapace P, Bucci F, Lasiecka I. Optimal boundary control and Riccati theory for abstract dynamics motivated by hybrid systems of PDEs. Adv Diff Equ 2005; 10(12): 1389-436.

[46] Bucci F, Lasiecka I. Optimal boundary control with critical penalization for a PDE model of fluid-solid interactions. Calc Var Partial Differ Equ 2010; 37 (1-2): 217-35

Received: March 15, 2013

Revised: May 02, 2013

Accepted: May 11, 2013

(C) Lasiecka et al.; Licensee Bentham Open.

This is an open access article licensed under the terms of the Creative Commons Attribution Non-Commercial License (http://creativecommons.org/licenses/by-nc/3.0/) which permits unrestricted, non-commercial use, distribution and reproduction in any medium, provided the work is properly cited. 